ESAIM: COCV 22 (2016) 236-266

DOI: $10.1051 / \mathrm{cocv} / 2015009$
ESAIM: Control, Optimisation and Calculus of Variations

www.esaim-cocv.org

\title{
ON THE ATTAINABLE SET FOR SCALAR BALANCE LAWS WITH DISTRIBUTED CONTROL *
}

\author{
Marco Corghi ${ }^{1}$ And Andrea Marson ${ }^{2}$
}

\begin{abstract}
The paper deals with the set of attainable profiles of a solution $u$ to a scalar balance law in one space dimension with strictly convex flux function

$$
\partial_{t} u+\partial_{x} f(u)=z(t, x) .
$$

Here the function $z$ is regarded as a bounded measurable control. We are interested in studying the set of attainable profiles at a fixed time $T>0$, both in case $z(t, \cdot)$ is supported in the all real line, and in case $z(t, \cdot)$ is supported in a compact interval $[a, b]$ independent on the time variable $t$.
\end{abstract}

Mathematics Subject Classification. 35L65, 35Q93.

Received April 28, 2014. Revised December 22, 2014.

Published online January 15, 2016.

\section{INTRODUCTION}

In this paper we consider a balance law in one space dimension, i.e.

$$
\partial_{t} u+\partial_{x} f(u)=z(t, x) \quad t \in[0, T], \quad x \in \mathbb{R},
$$

augmented with an initial datum

$$
u(0, x)=u_{0}(x), \quad x \in \mathbb{R} .
$$

Here $u_{0} \in L^{1}(\mathbb{R}) \cap L^{\infty}(\mathbb{R})$ is given, the flux $f$ is a $\mathcal{C}^{2}$ function which is assumed to be uniformly strictly convex, i.e. there holds

$$
\inf _{u \in \mathbb{R}} f^{\prime \prime}(u)>0
$$

and the source term $z \in L^{\infty}(] 0, T[\times \mathbb{R})$ is regarded as a control. We are interested in studying the set of attainable profiles at time $T$, i.e.

$$
\mathcal{A}(T, \mathcal{Z}) \doteq\left\{v \in L^{\infty}(\mathbb{R}): \exists z \in \mathcal{Z} \text { and a solution } u \text { to }(1.1)-(1.2): u(T, x)=v(x) \text { a.e. }\right\},
$$

Keywords and phrases. Conservation laws, distributed control.

* This research was partially supported by the UE project ERC Starting Grant CONLAWS Systems of Hyperbolic Conservation Laws: Singular Limits, Properties of Solutions and Control Problems, by the Italian Ministry of University and Research PRIN project Systems of Conservation Laws and Fluid Dynamics: Methods and Applications, and by CARIPARO Project NPDE Nonlinear Partial Differential Equations: models, analysis, and control-theoretic problems.

1 Via Monte Sabotino 99, 41124 Modena, Italy. mcorghi@gmail.com

2 Dipartimento di Matematica, Via Trieste 63, 35121 Padova, Italy. marson@math.unipd.it 
where $\mathcal{Z} \subseteq L^{\infty}(] 0, T[\times \mathbb{R})$ is a given set of admissible controls. In particular, we will focus our attention to the cases

(1) $\mathcal{Z}=L^{\infty}(] 0, T[\times \mathbb{R})$;

(2) if $a, b \in \mathbb{R}, a<b$, are given, then

$$
\mathcal{Z}=\mathcal{Z}_{a, b} \doteq\left\{z \in L^{\infty}(] 0, T[\times \mathbb{R}): \operatorname{supp} z(t, \cdot) \subseteq[a, b] \forall t \in\right] 0, T[\},
$$

where $\operatorname{supp} z(t, \cdot)$ denotes the essential support of the function $x \mapsto z(t, x)$.

It is well known (see $[7,13])$ that balance laws, in general, do not admit globally defined Lipschitz continuous solutions, even in presence of smooth initial data. Hence, we will consider a framework of entropy weak solutions (see Def. 2.1 below), that may well suffer from jump discontinuities.

Since the pioneering papers $[5,17]$, control problems for balance laws have received an increasing attention. Regarding scalar conservation/balance laws, several papers appeared in recent year, e.g. see [11, 20-22]. In particular, in [11] the author studied the attainability of $\mathcal{C}^{1}$ functions for classical solutions of a balance law (1.1) in a bounded domain, say $[0, T] \times[0,1]$, when the distributed control $z$ depends only on the time variable $t$. In $[20]$ the author dealt with a conservation law

$$
\partial_{t} u+\partial_{x} f(u)=0
$$

with a flux function $f$ with finitely many inflection points. The equation (1.6) is considered in a bounded domain $[0, T] \times[0,1]$ and the initial datum $u_{0}$ at $(1.2)$ is assumed to belong to $L^{\infty}(0,1)$. The boundary data at $x=0$ and $x=1$ are regarded as controls, and it is proved the existence of controls steering the solution to (1.2)-(1.6) to a constant state in a time $T$ depending on the $L^{\infty}$-norm of $u_{0}$. Both the papers $[11,20]$ use the return method by Coron ([9], Chap. 6). In particular, in [20] a viscous approximation of a conservation law is considered, i.e.

$$
\partial_{t} u+\partial_{x} f(u)=\varepsilon \partial_{x x}^{2} u,
$$

and a controllability result for the inviscid equation is obtained by letting $\varepsilon \rightarrow 0$. Instead, a distributed control is considered in [14] for a viscous Burgers equation with Dirichlet conditions in the interval $[0,1]$. Here the authors give a precise estimate of the minimal time needed in order to drive to zero a given initial datum. In [21] the author studied the attainable set at time $t=T$ in a bounded domain $[0, T] \times[0,1]$, but in a context of weak entropy solutions. Again, the author assumes to control the boundary data at $x=0$ and $x=1$, and the source term, which is supposed to depend only on the time variable $t$. In [22] the same author obtained a stabilization result around a given constant state in the same context of [21], but using a feedback control, which is explicitly given.

Regarding systems of conservation/balance laws, to our knowledge only few papers appeared. In [3, 4], the authors studied the attainable set for boundary controllable systems of conservation laws in the Temple class [13]. Other papers deal with controllability around a constant state $[10,15,16]$, non controllability result [8], or asymptotic stabilizability to a constant state [6].

In this paper we are interested in determining a sufficiently large subset of the set $\mathcal{A}(T, \mathcal{Z})$ at $(1.4)$, with $\mathcal{Z}=L^{\infty}(] 0, T[\times \mathbb{R})$ and $\mathcal{Z}=\mathcal{Z}_{a, b}$. In particular, such a set is expected to contain bounded functions suffering jump discontinuities, and fulfilling an upper bound on the positive waves (and hence on the positive total variation),

$$
\sup _{x \in \mathbb{R}} \limsup _{h \rightarrow 0} \frac{v(x+h)-v(x)}{h}<+\infty
$$

where $v \in L^{1}(\mathbb{R}) \cap \mathrm{BV}_{\text {loc }}(\mathbb{R})$ is given. Such a condition comes from Oleinik's type estimate ([13], Chap. 8) on the decay of positive waves. This will suffice to find a bounded control $z$ supported in $] 0, T[\times \mathbb{R}$ which drives $u_{0}$ to $v$ in time $T$. Instead, if $\operatorname{supp} z \subseteq[0, T] \times[a, b]$, together with (1.7) more conditions will be needed on the behavior of $v$ outside $[a, b]$. 
We will proceed by constructing at the same time a control $z$ and a solution $u$ to (1.1)-(1.2) with the desired properties. In case $\mathcal{Z}=L^{\infty}(] 0, T[\times \mathbb{R})$, such a construction is performed by means of a suitably modified front-tracking algorithm $[7,18]$, where the approximate solutions are piecewise Lipschitz continuous. This will allow us to construct a bounded control $z$ and a weak entropy solution to (1.1)-(1.2) steering $u_{0}$ to zero. Then, we will drive $u=0$ to a final profile $v$ by first solving backward (1.6) in a small time interval by means of a locally Lipschitz function $w=w(t, x)$, and then gluing $u=0$ and $w$ with a suitable control $z$. Instead, in case $\mathcal{Z}=\mathcal{Z}_{a, b}$, we study the attainable set $\mathcal{A}\left(T, \mathcal{Z}_{a, b}\right)$ for solutions to (1.1) with null initial datum, and hence we take $u_{0}(x) \equiv 0$ at (1.2). Inspired by [5], we will heavily use the theory of generalized characteristics by Dafermos [12], in particular in order to reconstruct a solution outside the bounded domain $[0, T] \times[a, b]$. Indeed, since $\operatorname{supp} z \subseteq[0, T] \times[a, b]$, a solution $u$ to (1.1) satisfies $(1.6)$ on $[0, T] \times(\mathbb{R} \backslash[a, b])$. This is a key point in order to understand how a compactly supported control $z$ affects a solution $u$ to (1.1) outside supp $z$.

The paper is organized as follows. In Section 2 we state the main Theorems and prove some preliminary Lemmas on a boundary value problem for a scalar conservation law. In Section 3 we prove Theorem 2.2 on the controllability for $\mathcal{Z}=L^{\infty}(] 0, T[\times \mathbb{R})$, while in Section 4 we consider the case $\mathcal{Z}=\mathcal{Z}_{a, b}$. Both these sections are self-contained and can be read separately; only the Lemma 3.3 is used in both. Moreover, they are divided in three subsections, each containing a step of the proofs. Finally, an Appendix is devoted to the proof of some technical results that are used in proving Theorem 2.2 in Section 3. We inserted several figures all along the paper in order to make the construction more clear.

\section{Preliminaries AND MAin RESUlts}

First of all, let us introduce some notations that will be used all along the paper. Given a function $v=v(x)$, $x \in \mathbb{R}$, we will adopt the usual notation

$$
v(\xi \pm)=\lim _{x \rightarrow \xi^{ \pm}} v(x),
$$

whenever the limits exist. Moreover, since $f$ is strictly convex, $f^{\prime}$ is strictly increasing, and hence it admits a smooth inverse, that will be denoted by $g$, i.e.

$$
g=\left(f^{\prime}\right)^{-1} .
$$

As it was pointed out in the introduction, in general a Cauchy's problem for a balance law does not admit a globally defined classical solution. Moreover, in order to select the physically relevant solution among the distributional ones, an admissibility criterion must be introduced $[7,13]$. Hence, we need the definition of weak entropy solution. For a more exhaustive treatment of such distributional solutions, we refer to $[7,13]$.

Definition 2.1. A function $u \in L^{\infty}([0, T] \times \mathbb{R})$ is a weak entropy solution to (1.1)-(1.2) if

(1) $[0, T] \ni t \mapsto u(t, \cdot) \in L_{l o c}^{1}(\mathbb{R})$ is continuous;

(2) $u(0, x)=u_{0}(x)$ for a.e. $x \in \mathbb{R}$;

(3) for all $\kappa \in \mathbb{R}$ there holds the entropy inequality

$$
\int_{0}^{T} \int_{\mathbb{R}}|u-\kappa| \partial_{t} \varphi+\operatorname{sgn}(u-\kappa)(f(u)-f(\kappa)) \partial_{x} \varphi \mathrm{d} x \mathrm{~d} t+\int_{0}^{T} \int_{\mathbb{R}} \operatorname{sgn}(u-\kappa) z(t, x) \varphi \mathrm{d} x \mathrm{~d} t \geq 0
$$

for all non negative $\varphi \in \mathcal{C}^{1}(] 0, T[\times \mathbb{R})$ with compact support.

The entropy inequality (2.2) was introduced by Kruzkov [19], and it can be replaced by an equivalent smooth version: in order $u$ to be an entropy weak solution to (1.1)-(1.2), it is required that for any convex entropy $\eta \in \mathcal{C}^{1}(\mathbb{R})$ with entropy flux $q$, i.e. $q^{\prime}(u)=f^{\prime}(u) \eta^{\prime}(u)$, there holds

$$
\int_{0}^{T} \int_{\mathbb{R}} \eta(u) \partial_{t} \varphi+q(u) \partial_{x} \varphi \mathrm{d} x \mathrm{~d} t+\int_{0}^{T} \int_{\mathbb{R}} \eta^{\prime}(u) z(t, x) \varphi \mathrm{d} x \mathrm{~d} t \geq 0
$$


for all nonnegative $\varphi \in \mathcal{C}^{1}(] 0, T[\times \mathbb{R})$ with compact support. In case $u=u(t, x)$ is a weak entropy solution to (1.1)-(1.2), then along each Lipschitz curve $x=\eta(t)$ in the $(t, x)$-plane along which $u$ suffers from a jump discontinuity, the Rankine-Hugoniot conditions hold,

$$
f(u(t, \eta(t)+))-f(u(t, \eta(t)-))=\dot{\eta}(t)[u(t, \eta(t)+)-u(t, \eta(t)-)],
$$

together with the admissibility conditions

$$
u(t, x-)>u(t, x+), \quad x=\eta(t),
$$

where, as usual, $u(t, \xi-)$ and $u(t, \xi+)$ are, respectively, the left and right limits of $u(t, \cdot)$ at $\xi$.

The Definition 2.1 allows to use the "doubling variables technique" introduced by Kruzkov [19], so that it can be proved that, if a weak entropy solution to (1.1)-(1.2) exists, then it is unique. Regarding a general existence theorem for (1.1)-(1.2), we refer to [19] again, while systems of balance laws, e.g., are treated in [1]. Moreover, to our knowledge, in literature smoothness assumptions on $z$ are usually required in order to get $a$ priori estimates on the positive waves [23]. Indeed, such a priori estimate is the main ingredient of the following

Theorem 2.2. Let $f \in \mathcal{C}^{2}(\mathbb{R})$ and (1.3) hold. Let $u_{0} \in L^{1}(\mathbb{R}) \cap L^{\infty}(\mathbb{R})$ and $T>0$ be given, and $v \in L^{1}(\mathbb{R}) \cap$ $\mathrm{BV}_{\text {loc }}(\mathbb{R})$ be such that $(1.7)$ holds. Then, $v \in \mathcal{A}\left(T, L^{\infty}(] 0, T[\times \mathbb{R})\right)$, where $\mathcal{A}(T, \mathcal{Z})$ is defined at (1.4). Hence, there exists $z \in L^{\infty}(] 0, T[\times \mathbb{R})$ such that the weak entropy solution u to $(1.1)-(1.2)$ satisfies $u(T, \cdot)=v$.

Observe that not any $L^{\infty}$ function $v$ can be attained. Indeed, there are a couple of obstructions. The first one is that the obvious idea of considering

$$
u(t, x)=u_{0}(x)+\frac{t}{T}\left(v(x)-u_{0}(x)\right)
$$

and next defining

$$
z \doteq \partial_{t} u+\partial_{x} f(u)
$$

where the derivatives are taken in distributional sense, does not work due to the regularity assumptions on $z$, which is assumed to be a bounded function. Conversely, such a method works well for Lipschitz continuous initial datum $u_{0}$ and final profile $v$. The second obstruction comes from the definition of weak entropy solution, and particularly from the admissibility condition (2.5) on the jump discontinuities of an admissible solution $u$. Such a condition forces a candidate attainable profile $v$ to suffer only from downward discontinuities, and not from upward ones.

Remark 2.3. We point out that condition (1.7) can be weakened to a local version. Indeed, if $v \in L^{1}(\mathbb{R}) \cap$ $\mathrm{BV}_{\text {loc }}(\mathbb{R})$ fulfills

$$
\sup _{x \in K} \limsup _{h \rightarrow 0} \frac{v(x+h)-v(x)}{h}<+\infty,
$$

for any compact set $K \subset \mathbb{R}$, we can still prove the existence of a bounded control $z$ steering $u_{0}$ to $v$ in time $T$, but at the price of technicalities, which we prefer to avoid. Indeed, the construction of a locally Lipschitz backward solution $u$ to $(1.6)$ with $u(T, \cdot)=v$ carried out at Lemma 4.1 can be performed locally in space thanks to $(2.6)$, and this suffice to prove that $v \in \mathcal{A}\left(T, L^{\infty}(] 0, T[\times \mathbb{R})\right)$. The only obstructions in getting $v$ by a bounded control $z$ with our method are

(1) whether $v$ has an upward jump, but in this case entropy conditions (2.5) would be violated, and hence $v$ can not be a weak entropy solution to (1.1), whatever $z$ is chosen;

(2) or

$$
\lim _{n \rightarrow+\infty} \limsup _{h \rightarrow 0} \frac{v\left(x_{n}+h\right)-v\left(x_{n}\right)}{h}=+\infty
$$

for a bounded sequence $\left\{x_{n}\right\}_{n \in \mathbb{N}} \subset \mathbb{R}$. 
It remains an open problem to establish if this last condition is a real obstruction to the attainability of the function $v$.

Next, we address the same problem, but dealing with compactly supported controls $z$. As stated in the Introduction, we consider an initial datum $u_{0}(x) \equiv 0$ for the Cauchy problem (1.1)-(1.2).

Theorem 2.4. Let $f \in \mathcal{C}^{2}(\mathbb{R})$ and (1.3) hold. Let $a, b \in \mathbb{R}, a<b$, and $T>0$ be given, and assume $u_{0}(x) \equiv 0$. Let $v \in \mathrm{BV}(\mathbb{R})$ satisfy the following conditions

(1) Condition (1.7) is fulfilled.

(2) If $x \notin[a, b]$, then

$$
\begin{aligned}
& x<a \quad \text { and } \quad v(x-) \neq 0 \quad \Longrightarrow \quad f^{\prime}(v(x-)) \leq \frac{x-a}{T}, \\
& x>b \quad \text { and } \quad v(x+) \neq 0 \quad \Longrightarrow \quad f^{\prime}(v(x+)) \geq \frac{x-b}{T},
\end{aligned}
$$

(3) Let $\alpha \leq a<b \leq \beta$ be such that

$$
\alpha=\sup \{x \leq a: v(y)=0 \forall y<x\}, \quad \beta=\inf \{x \geq b: v(y)=0 \forall y>x\} .
$$

Then

$$
\begin{aligned}
& v(\alpha+) \neq 0 \quad \Longrightarrow \quad\left\{\begin{array}{lll}
f^{\prime}(v(\alpha+))<\frac{\alpha-a}{T} & \text { if } \quad \alpha<a, \\
f^{\prime}(v(\alpha+)) \leq 0 & \text { if } \quad \alpha=a,
\end{array}\right. \\
& v(\beta-) \neq 0 \quad \Longrightarrow \quad \begin{cases}f^{\prime}(v(\beta-))>\frac{\beta-b}{T} & \text { if } \beta>b, \\
f^{\prime}(v(\beta-)) \geq 0 & \text { if } \quad \beta=b .\end{cases}
\end{aligned}
$$

(4) There hold

$$
\begin{aligned}
& \sup \left\{\limsup _{h \rightarrow 0} \frac{v(x+h)-v(x)}{h}-\frac{f^{\prime}(v(x))}{(x-a) f^{\prime \prime}(v(x))}: x<a, v(x) \neq 0\right\}<0, \\
& \sup \left\{\limsup _{h \rightarrow 0} \frac{v(x+h)-v(x)}{h}-\frac{f^{\prime}(v(x))}{(x-b) f^{\prime \prime}(v(x))}: x>b, v(x) \neq 0\right\}<0 .
\end{aligned}
$$

Then $v \in \mathcal{A}\left(T, \mathcal{Z}_{a, b}\right)$, where $\mathcal{A}(T, \mathcal{Z})$ is defined at (1.4), and $\mathcal{Z}_{a, b}$ is defined at (1.5). Hence, there exists $z \in \mathcal{Z}_{a, b}$ such that the weak entropy solution $u$ to (1.1) and (1.2) with $u_{0}(x) \equiv 0$ satisfies $u(T, \cdot)=v$.

Remark 2.5. Observe that, using conditions (2.7) and (2.8) and the fact that $v$ is a bounded function, there exist $x_{a}, x_{b} \in \mathbb{R}, x_{a}<x_{b}$, such that $v(x)=0$ for all $x \notin\left[x_{a}, x_{b}\right]$. There follows that $\alpha$ and $\beta$ at $(2.9)$ are well defined real numbers. Moreover, condition (1.7) ensures that, if $v$ suffers a jump discontinuity at $x$, then $v(x-)>v(x+)$. Due to the monotonicity property of $f^{\prime}$, it follows that, if $v(x+) \neq 0$ with $x<a$, then thanks to $(2.7)$

$$
f^{\prime}(v(x+)) \leq f^{\prime}(v(x-)) \leq \frac{x-a}{T} .
$$

Conversely, if $x>b$ we get

$$
f^{\prime}(v(x-)) \geq f^{\prime}(v(x+)) \geq \frac{x-b}{T} .
$$


Using the theory of generalized characteristics [12] we can give a geometric interpretation of conditions (2.7)-(2.13). In particular, conditions from (2.7) through (2.11) turn out to be also necessary for a final profile $v=v(x)$ to be attainable with a bounded control $z=z(t, x)$ supported in $[0, T] \times[a, b]$. Indeed, choose any bounded function $z=z(t, x)$ such that $z(t, x)=0$ whenever $x \notin[a, b]$. Let $u$ be an entropy solution to (1.1)-(1.2) with $u_{0} \equiv 0$ and fulfilling $u(t, x)=v(x)$ for a.e. $x \in \mathbb{R}$. Then $u$ is a weak entropy solution to

$$
\partial_{t} u+\partial_{x} f(u)=0, \quad t \in[0, T], x<a \text { or } x>b .
$$

Once a point $(T, \xi)$ is fixed, say $\xi<a$, then the minimal and maximal backward characteristic from $(T, \xi)$, whose equations are

$$
x=\xi+f^{\prime}(v(\xi-))(t-T), \quad x=\xi+f^{\prime}(v(\xi+))(t-T),
$$

are genuine (true) characteristic. In particular, $u$ is constant along such lines, and hence choosing $t=0$, we get

$$
v(\xi-)=u_{0}\left(\xi-f^{\prime}(v(\xi-)) T\right), \quad v(\xi+)=u_{0}\left(\xi-f^{\prime}(v(\xi+)) T\right),
$$

so that, whenever

$$
\xi<a, \quad f^{\prime}(v(\xi-))>\frac{\xi-a}{T},
$$

there holds

$$
v(\xi-)=u(\xi-)=u_{0}\left(\xi-T f^{\prime}(v(\xi-))\right)=0,
$$

and similarly for $\xi>b$.

Conditions (2.10) and (2.11) are related to configurations where we expect to have a shock discontinuity at $x=\alpha$ or at $x=\beta$ at time $t=T$. In such a case the maximal (resp. minimal) characteristic from $(T, \alpha)$ (resp. $(T, \beta))$ must satisfy

$$
\begin{gathered}
\alpha+\left.f^{\prime}(v(\alpha+))(t-T)\right|_{t=0}=\alpha-f^{\prime}(v(\alpha+)) T>a \\
\left(\operatorname{resp} . \beta+\left.f^{\prime}(v(\beta+))(t-T)\right|_{t=0}=\beta-f^{\prime}(v(\beta+)) T<b\right),
\end{gathered}
$$

otherwise, with calculations similar to the previous ones, we find $v(\alpha+)=0$ (and, respectively, $v(\beta-)=0$ ).

Regarding conditions (2.12) and (2.13), observe that, since (2.14) holds, minimal and maximal backward characteristics cannot intersect in the regions $] 0, T] \times]-\infty, a[$ and $] 0, T] \times] b,+\infty[$ (see again [12]). If $(2.12$ ) and (2.13) hold for a final profile $v$, then we can prove that the candidate minimal and maximal backward characteristics (2.15) from any point $(T, \xi), \xi<a$ or $\xi>b$, enjoy this property. This allows us to construct a desired bounded control $z$ and an entropy solution $u$ to $(1.1)-(1.2)$, with $u_{0}(x) \equiv 0$, fulfilling $u(T, \cdot)=v$ (see the proof of Prop. 4.5 at Sect. 4.2).

\subsection{Technical lemmas}

The lemma that follows will be used in the proof of Theorem 2.4 (see Lem. 4.1). The proof of the lemma is entirely similar to the proof of ([5], Prop. 3.1), thus we will omit it.

Lemma 2.6. Let $\varpi<0$ and $\alpha<a$ be given. Assume that

$$
f^{\prime}(0) \geq 0, \quad f^{\prime}(\varpi)<(\alpha-a) / T .
$$

Call

$$
\tau_{\varpi}=T-\frac{\alpha-a}{f^{\prime}(\varpi)},
$$


so that $0<\tau_{\varpi}<T$. Fix $\left.\tau \in\right] 0, \tau_{\varpi}[$. Then, there exists a Lipschitz function $\phi:[\tau, T] \rightarrow \mathbb{R}$, such that the solution $w=w(t, x)$ to the initial boundary value problem

$$
\left\{\begin{array}{l}
\partial_{t} w+\partial_{x} f(w)=0 \\
w(0, x)=0 \\
w(t, a)=\left\{\begin{array}{ll}
0 & \text { if } 0 \leq t<\tau, \\
\phi(t) & \text { if } \quad \tau \leq t \leq T,
\end{array} \quad t \in[0, T], \quad x<a,\right.
\end{array}\right.
$$

satisfies

$$
w(T, x)=\left\{\begin{array}{lll}
0 & \text { if } \quad x<\alpha, \\
\varpi & \text { if } \quad \alpha \leq x<a .
\end{array}\right.
$$

Moreover, the solution $w$ is piecewise Lipschitz, and suffering only one shock departing from $x=a$ at time $t=\tau$, and reaching $x=\alpha$ at time $t=T$.

Remark 2.7. Observe that $\tau_{\varpi}$ is the time at which the candidate maximal backward characteristic from $(T, \alpha)$ reaches $x=a$. As regard as the function $\phi$, as it can be easily deduced from the proof of ([5], Prop. 3.1), we can choose the function $\phi$ at Lemma 2.6 so that $\phi(t)<0$ for any $t \in[\tau, T]$, and $\phi(t)=\varpi$ whenever $t \in\left[\tau_{\varpi}, T\right]$. Moreover, we can choose $\phi(\tau)$ so that

$$
|\phi(\tau)| \leq C_{\varpi, \alpha},
$$

with $C_{\varpi, \alpha}>0$ depending only on $\varpi$ and $\alpha$.

Next lemma, whose proof will be given, enters in proof of Theorem 2.4 by means of Lemma 4.2.

Lemma 2.8. Let $\varpi^{\prime}<\varpi<0$ and $\left.\tau \in\right] 0, T[$ be given, with

$$
\frac{f\left(\varpi^{\prime}\right)-f(0)}{\varpi^{\prime}}<0, \quad f^{\prime}(\varpi)=0 .
$$

Then, there exist $\left.\widetilde{\tau}_{\varpi} \in\right] \tau, T[$ and a Lipschitz function $\phi:[\tau, T] \rightarrow \mathbb{R}$ such that

$$
\begin{gathered}
\phi(t)<0 \quad \forall t \in[\tau, T], \quad \phi(\tau)=\varpi^{\prime}, \\
\phi(t)=\varpi \quad \forall t \in\left[\widetilde{\tau}_{\varpi}, T\right],
\end{gathered}
$$

and the solution $w=w(t, x)$ to the initial boundary value problem (2.17) is piecewise Lipschitz continuous with $w(T, x)=0$ for $x<a$. Moreover, $w$ suffers only one shock discontinuity departing from $x=a$ at time $t=\tau$, and reaching $x=a$ at time $t=T$.

Proof. Let

$$
\xi=a+\frac{f\left(\varpi^{\prime}\right)-f(0)}{\varpi^{\prime}}(T-\tau)
$$

and choose $\widetilde{\tau}_{\varpi}$ so that (see Fig. 1)

$$
T+\frac{a-\xi}{f^{\prime}\left(\varpi^{\prime}\right)}<\widetilde{\tau}_{\varpi}<T .
$$

We let $\tau \leq \tau_{1}<\tau_{2} \leq \widetilde{\tau}_{\varpi}$ and call

$$
\phi_{\tau_{1}, \tau_{2}}(t)=\left\{\begin{array}{lll}
\varpi^{\prime} & \text { if } \quad \tau \leq t<\tau_{1}, \\
g\left(f^{\prime}\left(\varpi^{\prime}\right) \frac{\tau_{2}-t}{\tau_{2}-\tau_{1}}\right) & \text { if } \quad \tau_{1} \leq t<\tau_{2}, \\
\varpi & \text { if } \quad \tau_{2} \leq t,
\end{array}\right.
$$




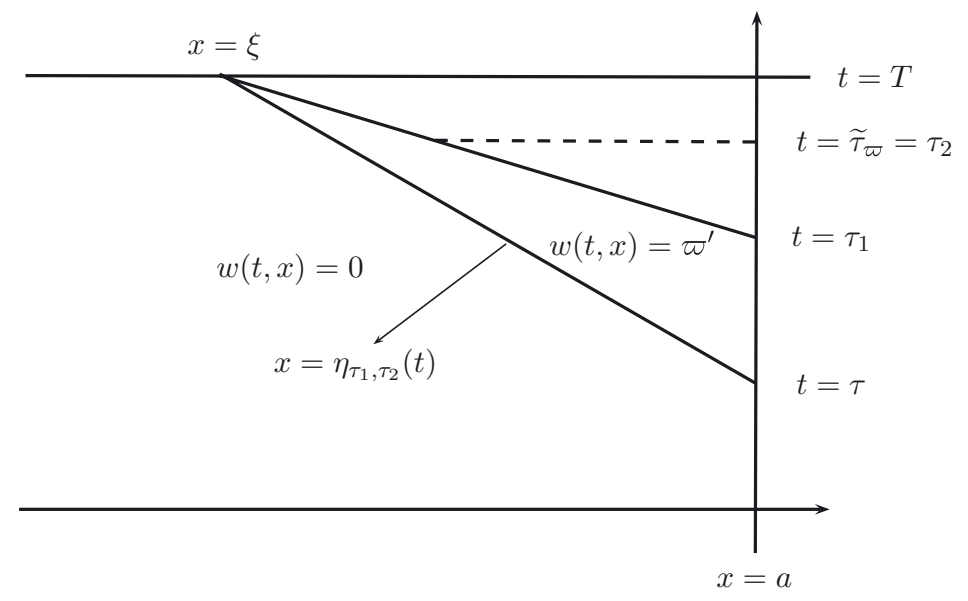

Figure 1 . The case $\eta_{\tau_{1}, \tau_{2}}(T)<a$.

where $g=\left(f^{\prime}\right)^{-1}$. Observe that for all $\tau \leq \tau_{1}<\tau_{2} \leq \widetilde{\tau}_{\varpi}$ the function $\phi_{\tau_{1}, \tau_{2}}$ is Lipschitz continuous, and satisfies conditions (2.19) with $\phi=\phi_{\tau_{1}, \tau_{2}}$. Moreover, due to the monotonicity properties of $f^{\prime}, \phi$ is increasing. Let $w_{\tau_{1}, \tau_{2}}=w_{\tau_{1}, \tau_{2}}(t, x)$ be the solution to

$$
\left\{\begin{array}{l}
\partial_{t} w+\partial_{x} f(w)=0 \\
w(0, x)=0 \\
w(t, a)=\left\{\begin{array}{lll}
0 & \text { if } 0 \leq t<\tau, \\
\phi_{\tau_{1}, \tau_{2}}(t) & \text { if } & \tau \leq t,
\end{array} \quad x<a .\right.
\end{array}\right.
$$

It turns out that $w_{\tau_{1}, \tau_{2}}$ is piecewise Lipschitz continuous and contains only one shock departing from $x=a$ at time $t=\tau$. Let $\left(t, \eta_{\tau_{1}, \tau_{2}}(t)\right), \eta_{\tau_{1}, \tau_{2}}(t) \leq a$, be the position of the shock at time $t$ in the $(t, x)$ plane. Moreover, we call (see Fig. 2)

$$
\zeta(t)=a+f^{\prime}\left(\varpi^{\prime}\right)\left(t-\tau_{1}\right), \quad t \geq \tau_{1},
$$

and set

$$
\Omega \doteq\left\{(t, x): \max \left\{\eta_{\tau_{1}, \tau_{2}}(t), \zeta(t)\right\}<x<a, t \geq \tau_{2}\right\}
$$

By using the method of characteristics, we get that

$$
w_{\tau_{1}, \tau_{2}}(t, x)=\phi_{\tau_{1}, \tau_{2}}(s(t, x)), \quad \eta_{\tau_{1}, \tau_{2}}(t)<x<a, t \geq \tau_{1},
$$

where

$$
s(t, x)= \begin{cases}t+\frac{a-x}{f^{\prime}\left(\varpi^{\prime}\right)} & \text { if } \quad \eta_{\tau_{1}, \tau_{2}}(t)<x<\zeta(t), \\ \frac{t+\tau_{2}-\sqrt{\Delta(t, x)}}{2} & \text { if } \quad \max \left\{\eta_{\tau_{1}, \tau_{2}}(t), \zeta(t)\right\}<x<a,\end{cases}
$$

with

$$
\Delta(t, x)=\left(t-\tau_{2}\right)^{2}-4 \frac{a-x}{f^{\prime}\left(\varpi^{\prime}\right)}\left(\tau_{2}-\tau_{1}\right) .
$$

We have

$$
w_{\tau_{1}, \tau_{2}}(t, x)=g\left(\frac{f^{\prime}\left(\varpi^{\prime}\right)}{2\left(\tau_{2}-\tau_{1}\right)}\left(\tau_{2}-t+\sqrt{\Delta(t, x)}\right)\right), \quad(t, x) \in \Omega,
$$




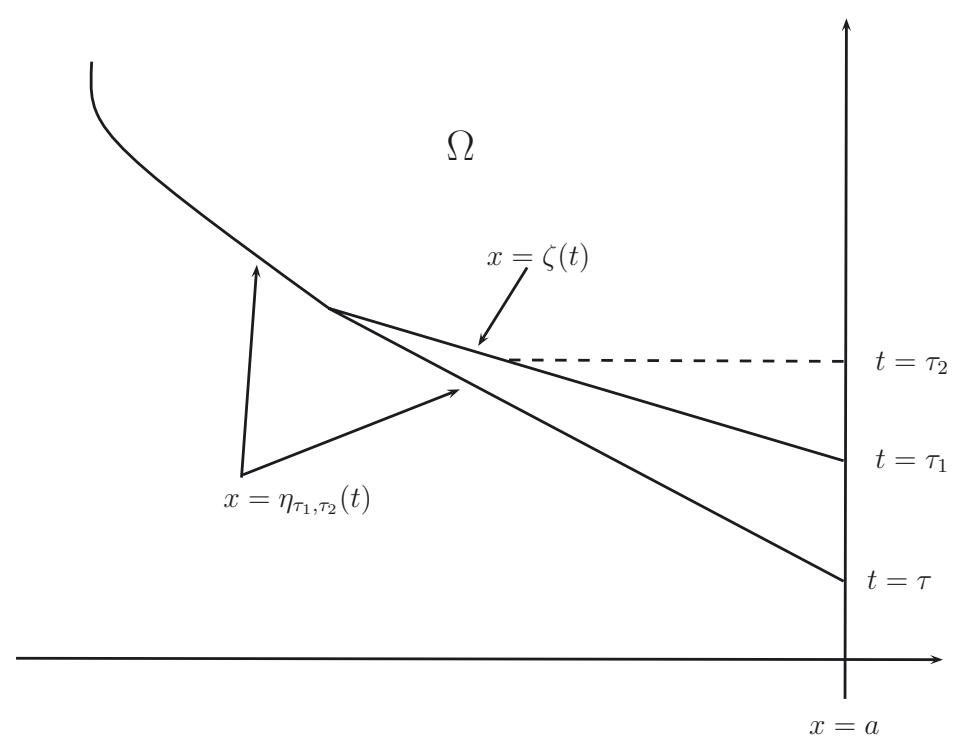

Figure 2. The set $\Omega$.

from which we get

$$
\lim _{\substack{x \rightarrow a^{-} \\(t, x) \in \Omega}} w_{\tau_{1}, \tau_{2}}(t, x)=\varpi .
$$

Hence, due to the entropy condition (2.5) and to the strict convexity of $f$, there exists $\bar{t}_{\tau_{1}, \tau_{2}} \geq \tau_{1}$ such that

$$
\eta_{\tau_{1}, \tau_{2}}\left(\bar{t}_{\tau_{1}, \tau_{2}}\right)=a .
$$

We will prove that there exists a choice of $\tau_{1}, \tau_{2}$, and hence of a boundary datum $\phi=\phi_{\tau_{1}, \tau_{2}}$, such that $\bar{t}_{\tau_{1}, \tau_{2}}=T$. First of all, let us prove that there exist $\tau_{1}, \tau_{2}$ such that $\eta_{\tau_{1}, \tau_{2}}(T)<a$, so that for the same choice of $\tau_{1}, \tau_{2}$ we have $\bar{t}_{\tau_{1}, \tau_{2}}>T$. Indeed, set

$$
\tau_{1}=T+\frac{a-\xi}{f^{\prime}\left(\varpi^{\prime}\right)}, \quad \tau_{2}=\widetilde{\tau}_{\varpi},
$$

with $\xi$ defined as at (2.20). Then

$$
\eta_{\tau_{1}, \tau_{2}}(t)=a+\frac{f\left(\varpi^{\prime}\right)-f(0)}{\varpi^{\prime}}(t-\tau), \quad \tau \leq t \leq T
$$

and

$$
w_{\tau_{1}, \tau_{2}}(t, x)=\left\{\begin{array}{lll}
0 & \text { if } \quad x<\eta_{\tau_{1}, \tau_{2}}(t), \\
\varpi^{\prime} & \text { if } \quad \eta_{\tau_{1}, \tau_{2}}(t)<x<\xi+f^{\prime}\left(\varpi^{\prime}\right)(t-T), \quad \tau \leq t \leq T,
\end{array}\right.
$$

so that $\eta_{\tau_{1}, \tau_{2}}(T)=\xi<a$ (see Fig. 1).

Now, let $\bar{t}_{\tau_{1}, \tau_{2}}$ satisfy $(2.24)$, and assume, by absurd, that $\bar{t}_{\tau_{1}, \tau_{2}} \geq T$ for any choice of $\tau_{1}, \tau_{2}$. Since (2.23), we have

$$
\lim _{x \rightarrow a^{-}} w_{\tau_{1}, \tau_{2}}(t, x)=\varpi \quad \forall \tau_{2} \leq t<\bar{t}_{\tau_{1}, \tau_{2}},
$$

and hence the speed of the shock $x=\eta_{\tau_{1}, \tau_{2}}(t)$ fulfills

$$
\dot{\eta}_{\tau_{1}, \tau_{2}}\left(\bar{t}_{\tau_{1}, \tau_{2}}\right)=\frac{f(\varpi)-f(0)}{\varpi}>0
$$


Now, assume that $\tau_{1}, \tau_{2} \rightarrow \tau$. Then $\phi_{\tau_{1}, \tau_{2}} \rightarrow \varpi$ in $L^{1}(0, T)$, and hence $w_{\tau_{1}, \tau_{2}} \rightarrow 0$ in $L_{l o c}^{1}([0, T] \times]-\infty, a[)$ (see [5], Thm. 4). Due to (2.25) and being $w_{\tau_{1}, \tau_{2}}$ Lipschitz continuous but along the shock curve $x=\eta_{\tau_{1}, \tau_{2}}(t)$, there exist $c, \delta>0$ independent on $\tau_{1}, \tau_{2}$ such that we have

$$
w_{\tau_{1}, \tau_{2}}(t, x) \leq-c \quad \forall(t, x): a+\frac{f(\varpi)-f(0)}{2 \varpi}\left(t-\bar{t}_{\tau_{1}, \tau_{2}}\right) \leq x<a, \quad \tau+\delta<t<\bar{t}_{\tau_{1}, \tau_{2}},
$$

contrary to the fact that $w_{\tau_{1}, \tau_{2}} \rightarrow 0$ in $L_{l o c}^{1}([0, T] \times]-\infty, a[)$. It follows that there exists $\tau<\tau_{1}<\tau_{2} \leq \widetilde{\tau}_{\varpi}$ such that $\bar{t}_{\tau_{1}, \tau_{2}}<T$.

Thanks to the continuous dependence of the solution to $(2.22)$ on the boundary datum, $\bar{t}_{\tau_{1}, \tau_{2}}$ depends continuously on $\tau_{1}, \tau_{2}$. It follows that there exist $\left.\left.\tau_{1}, \tau_{2} \in\right] \tau, \widetilde{\tau}_{\varpi]}\right]$ such that $\bar{t}_{\tau_{1}, \tau_{2}}=T$, and hence $\eta_{\tau_{1}, \tau_{2}}(T)=a$, thus proving the lemma.

\section{Proof of Theorem 2.2}

The proof of Theorem 2.2 is performed in three steps.

(1) First of all, in Lemma 3.1 we construct an $L^{\infty}$ control $z$ that drives the initial datum $u_{0}$ to zero in time $t=T / 2$. This is done by introducing suitable approximate solutions to a balance laws by means of an adapted front tracking algorithm (see $[7,18]$ and the references therein).

(2) Next, we take into consideration the final profile $v=v(x)$ at $t=T$. Using condition 1.7, we are able to trace back the candidate generalized characteristics [12], and find $\tau_{v}>0$ and a function $w=w(t, x)$, $T-\tau_{v} \leq t \leq T$, locally Lipschitz continuous for $t<T$, which is a solution to

$$
\partial_{t} w+\partial_{x} f(w)=0
$$

that fulfills $w(T, x)=v(x)$ for a.e. $x$ (see Lem. 3.3).

(3) Finally, in the time interval $\left[T / 2, T-\tau_{v}\right]$ we glue together the solutions and the controls at Steps 1 and 2. This is done by means of Lemma 3.4, whose proof is straightforward.

\subsection{Step 1 - Driving $\mathbf{u}_{0}$ to zero}

Let's start with the first step, which is the more technical.

Lemma 3.1. Let $u_{0} \in L^{1}(\mathbb{R}) \cap L^{\infty}(\mathbb{R})$ be given. Then there exists $\zeta^{0} \in L^{\infty}(] 0, T / 2[\times \mathbb{R})$ such that the solution $u$ to (1.1)-(1.2) with $z(t, x)=\zeta^{0}(t, x)$ fulfills $u(T / 2, x) \equiv 0$.

Proof. We will obtain $u$ and $\zeta^{0}$ as limits of suitable approximations, say $u^{\varepsilon}=u^{\varepsilon}(t, x)$ and $\zeta^{\varepsilon}=\zeta^{\varepsilon}(t, x)$ respectively, $\varepsilon \rightarrow 0^{+}$. First of all, without loss of generality we can assume that $u_{0}$ has bounded total variation. Indeed, if not, we can take $\zeta^{0}(t, x) \equiv 0$ for $t \in[0,2 \delta[, \delta>0$ suitably small, and consider the solution $u$ to

$$
\left\{\begin{array}{l}
\partial_{t} u+\partial_{x} f(u)=0 \\
u(0, x)=u_{0}(x),
\end{array} \quad 0 \leq t<2 \delta .\right.
$$

Such a solution satisfies the classical Oleinik's condition ([13], Chap. 8), and hence $u(t, \cdot) \in \mathrm{BV}(\mathbb{R})$ for any $t \in] 0,2 \delta\left[\right.$. Hence, we can take $u(\delta, \cdot)$ as a new BV initial datum. Now, let $u_{0}^{\varepsilon}, \varepsilon>0$, be a piecewise constant approximation of $u_{0}$ such that

$$
\text { Tot.Var. } u_{0}^{\varepsilon} \leq \text { Tot.Var. } u_{0}, \quad\left\|u_{0}^{\varepsilon}-u_{0}\right\|_{L^{1}(\mathbb{R})} \leq \varepsilon .
$$

We may assume that $u_{0}^{\varepsilon}$ as a finite number of points of jump, say $x_{1}<x_{2}<\ldots<x_{n}$ be the points of jump of $u_{0}^{\varepsilon}$. Denote by $u_{0}^{\varepsilon}\left(x_{i}-\right)$ and $u_{0}^{\varepsilon}\left(x_{i}+\right)$ the left and right limits of $u_{0}^{\varepsilon}$ at $x_{i}$, respectively, i.e.

$$
u_{0}^{\varepsilon}\left(x_{i} \pm\right) \doteq \lim _{x \rightarrow x_{i}^{ \pm}} u_{0}^{\varepsilon}(x)
$$


Moreover, without loss of generality, we assume that

$$
u_{0}^{\varepsilon}\left(x_{i}+\right)>u_{0}^{\varepsilon}\left(x_{i}-\right) \quad \Longrightarrow \quad\left|u_{0}^{\varepsilon}\left(x_{i}+\right)-u_{0}^{\varepsilon}\left(x_{i}-\right)\right|<\varepsilon .
$$

Next, we let

$$
\begin{aligned}
U_{i}^{\varepsilon+}(t) & \doteq \frac{T-2 t}{T} u_{0}^{\varepsilon}\left(x_{i}+\right), \\
U_{i}^{\varepsilon-}(t) & \doteq \frac{T-2 t}{T} u_{0}^{\varepsilon}\left(x_{i}-\right),
\end{aligned}
$$

Observe that, being $u_{0}^{\varepsilon}\left(x_{i}+\right)=u_{0}^{\varepsilon}\left(x_{i+1}-\right)$ and $u_{0}^{\varepsilon} \in L^{1}(\mathbb{R})$, we can define

$$
\begin{aligned}
& U_{i}^{\varepsilon}(t) \doteq U_{i}^{\varepsilon+}(t)=U_{i+1}^{\varepsilon-}(t), \quad i=1, \ldots, n-1 \\
& U_{0}(t) \doteq U_{1}^{\varepsilon-}(t) \equiv 0, \quad U_{n}(t) \doteq U_{n}^{\varepsilon+}(t) \equiv 0 .
\end{aligned}
$$

Now we define $n$ curves $\xi_{i}=\xi_{i}(t), i=1, \ldots, n$, which may not be distinct in some non degenerate interval, and which will be the candidate discontinuity lines in the approximate solution $u^{\varepsilon}=u^{\varepsilon}(t, x)$ that we are constructing. At first, we let $\xi_{i}=\xi_{i}(t), i=1, \ldots, n$, be the solution to the Cauchy's problem

$$
\left\{\begin{array}{l}
\dot{\xi}_{i}(t)=\frac{f\left(U_{i}^{\varepsilon}(t)\right)-f\left(U_{i-1}^{\varepsilon}(t)\right)}{U_{i}^{\varepsilon}(t)-U_{i-1}^{\varepsilon}(t)} \\
\xi_{i}(0)=x_{i}
\end{array}\right.
$$

Next, whenever two (or more) of these curves collide at time $t=\tau$, say $\xi_{i}, \xi_{i+1}, \ldots \xi_{i+p}, p \geq 1$, for $t>\tau$ we let $\xi_{i}(t)=\xi_{i+1}=\ldots=\xi_{i+p}(t) \doteq \xi(t)$ be the solution to the Cauchy's problem

$$
\left\{\begin{array}{l}
\dot{\xi}(t)=\frac{f\left(U_{i+p}^{\varepsilon}(t)\right)-f\left(U_{i-1}^{\varepsilon}(t)\right)}{U_{i+p}^{\varepsilon}(t)-U_{i-1}^{\varepsilon}(t)} \\
\left.\xi(\tau)=\xi_{i}(\tau)=\ldots=\xi_{i+p}(\tau)\right)
\end{array}\right.
$$

In this way the condition

$$
\xi_{i}(t) \leq \xi_{i+1}(t) \quad \forall t \in[0, T / 2], \quad i=1, \ldots, n-1
$$

is always fulfilled (see Fig. 3). In any case, since $f$ is strictly convex, there holds

$$
\xi_{i}, \xi_{i+1} \text { collide at time } \tau \Longrightarrow \min \left\{U_{i+1}^{\varepsilon}(\tau-)-U_{i}^{\varepsilon}(\tau-), U_{i}^{\varepsilon}(\tau-)-U_{i-1}^{\varepsilon}(\tau-)\right\}<0,
$$

i.e. at least one of the quantities $U_{i+1}^{\varepsilon}(\tau-)-U_{i}^{\varepsilon}(\tau-)$ and $U_{i}^{\varepsilon}(\tau-)-U_{i-1}^{\varepsilon}(\tau-)$ is negative. In this way the strip $[0, T / 2] \times \mathbb{R}$ is divided in a finite number of connected components $\Omega_{0}, \ldots, \Omega_{n}$ defined by (see Fig. 3 )

$$
\begin{aligned}
\Omega_{0} & =\left\{(t, x) \in[0, T / 2] \times \mathbb{R}: x<\xi_{1}(t)\right\}, \\
\Omega_{i} & =\left\{(t, x) \in[0, T / 2]: \xi_{i}(t) \leq x<\xi_{i+1}(t)\right\}, \quad i=1, \ldots, n-1, \\
\Omega_{n} & =\left\{(t, x) \in[0, T / 2]: x \geq \xi_{n}(t)\right\} .
\end{aligned}
$$

Now, we define $u^{\varepsilon}=u^{\varepsilon}(t, x)$ as follows

$$
u^{\varepsilon}(t, x)= \begin{cases}U_{i}^{\varepsilon}(t) & \text { if } \quad(t, x) \in \Omega_{i}, i=1, \ldots, n-1, \\ 0 & \text { if } \quad(t, x) \in \Omega_{0} \cup \Omega_{n},\end{cases}
$$

so that $u^{\varepsilon}$ is piecewise continuous, with jumps along the Lipschitz's curves $\xi_{1}, \ldots, \xi_{n}$ such that

$$
\Delta u^{\varepsilon}\left(t, \xi_{i}(t)\right)=U_{i}^{\varepsilon}(t)-U_{i-1}^{\varepsilon}(t),
$$




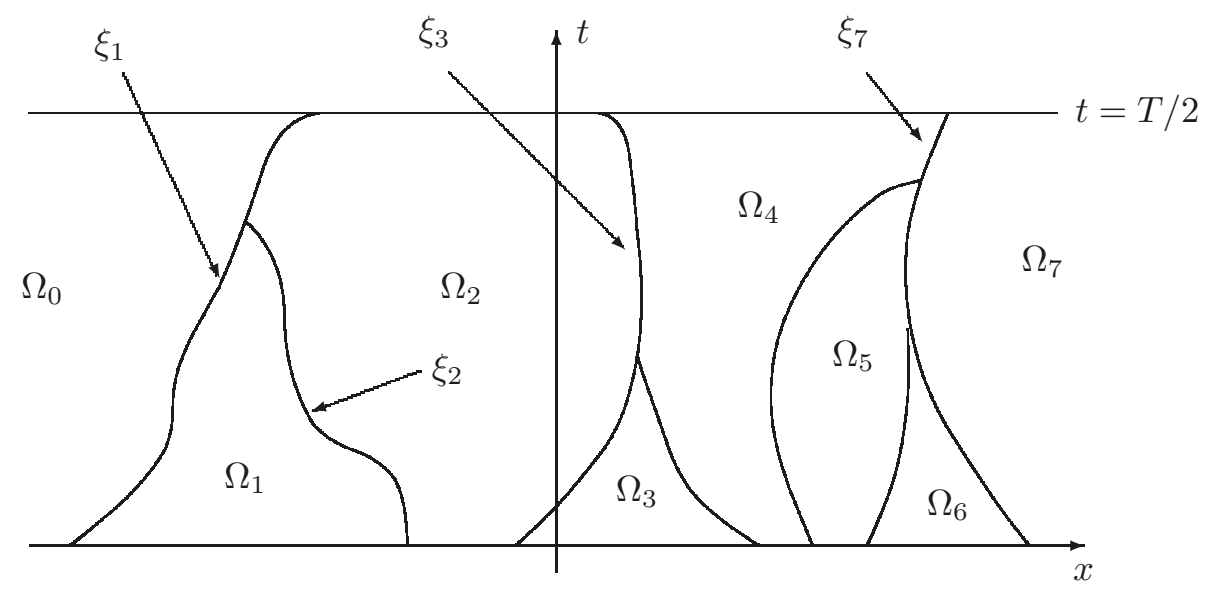

FiguRE 3 . The connected components $\Omega_{i}$.

and, due to (3.1) and (3.6),

$$
\Delta u^{\varepsilon}\left(t, \xi_{i}(t)\right)>0 \quad \Longrightarrow \quad \Delta u^{\varepsilon}\left(t, \xi_{i}(t)\right)<\frac{T-2 t}{T} \varepsilon<\varepsilon .
$$

It turns out that in the approximate solution $u^{\varepsilon}$ the jumps corresponding to a rarefaction wave, $\Delta u^{\varepsilon}\left(t, \xi_{i}(t)\right)>0$, have size at most $\varepsilon$. Regarding $\zeta^{\varepsilon}=\zeta^{\varepsilon}(t, x)$, its definition goes as follows

$$
\zeta^{\varepsilon}(t, x) \doteq \partial_{t} u^{\varepsilon}(t, x)+\partial_{x} f\left(u^{\varepsilon}(t, x)\right)= \begin{cases}-\frac{2}{T} u_{0}^{\varepsilon}\left(x_{i}+\right) & \text { if } \quad(t, x) \in \Omega_{i}, i=1, \ldots, n-1, \\ 0 & \text { if } \quad(t, x) \in \Omega_{0} \cup \Omega_{n} .\end{cases}
$$

With definitions (3.7)-(3.9) and due to (3.4) and (3.8), $[0, T / 2] \ni t \mapsto u^{\varepsilon}(t, \cdot) \in L^{1}(\mathbb{R})$ is continuous, $u^{\varepsilon}(T / 2, x) \equiv 0$ for all $\varepsilon>0$, and

$$
\begin{aligned}
\int_{0}^{T / 2} \int_{\mathbb{R}}\left|u^{\varepsilon}-\kappa\right| \partial_{t} \varphi+\operatorname{sgn}\left(u^{\varepsilon}-\kappa\right)\left(f\left(u^{\varepsilon}\right)-\right. & f(\kappa)) \partial_{x} \varphi \mathrm{d} x \mathrm{~d} t \\
& +\int_{0}^{T / 2} \int_{\mathbb{R}} \operatorname{sgn}\left(u^{\varepsilon}-\kappa\right) \zeta^{\varepsilon}(t, x) \varphi \mathrm{d} x \mathrm{~d} t \geq \mathcal{O}(1) \cdot \varepsilon \cdot \text { Tot.Var. } u_{0},
\end{aligned}
$$

holds for any nonnegative $\varphi \in \mathcal{C}^{1}(] 0, T / 2[\times \mathbb{R})$ with compact support, where $\mathcal{O}(1)$ is the usual Landau's symbol (e.g., see [7], Chap. 7 or [13], Chap. 14). Since by construction we have

$$
\text { Tot.Var. } u^{\varepsilon}(t, \cdot) \leq \text { Tot.Var. } u_{0}, \quad \text { Tot.Var. } \zeta^{\varepsilon}(t, \cdot) \leq \frac{2}{T} \text { Tot.Var. } u_{0}
$$

for all $t \in[0, T / 2]$, using Helly's theorem we get a sequence $\left\{\varepsilon_{n}\right\}_{n \geq 1}, \varepsilon_{n}>0$, such that $\varepsilon_{n} \rightarrow 0$ and $\left\{u^{\varepsilon_{n}}\right\}_{n \geq 1}$ and $\left\{\zeta^{\varepsilon_{n}}\right\}_{n \geq 1}$ converge in $L_{l o c}^{1}(] 0, T / 2[\times \mathbb{R})$, to bounded functions $u$ and $\zeta^{0}$, respectively, such that

Tot.Var. $u(t, \cdot) \leq$ Tot.Var. $u_{0}, \quad$ Tot.Var. $\zeta^{0}(t, \cdot) \leq \frac{2}{T}$ Tot.Var. $u_{0} \quad \forall t \in[0, T / 2]$.

Moreover, $[0, T / 2] \ni t \mapsto u(t, \cdot)$ is continuous w.r.t. the $L_{l o c}^{1}$ topology, and $u(0, \cdot)=u_{0}$ and $u(T / 2, \cdot)=0$. Now, observe that $u$ is a distributional solution of (1.1) and (1.2) in $] 0, T / 2[\times \mathbb{R}$, i.e.

$$
\int_{0}^{T / 2} \int_{\mathbb{R}} u \partial_{t} \phi+f(u) \partial_{x} \phi \mathrm{d} x \mathrm{~d} t+\int_{0}^{T / 2} \int_{\mathbb{R}} \zeta^{0} \phi \mathrm{d} x \mathrm{~d} t=0,
$$


for any $\phi \in \mathcal{C}^{1}(] 0, T / 2[\times \mathbb{R})$ with compact support. It follows that, since $x \mapsto f(u(t, x))$ has bounded total variation, uniformly in $t$ (see [2], Thm. 3.96), $u \in \mathrm{BV}([0, T / 2] \times \mathbb{R})$. Hence, due to Corollary A.4 in the Appendix,

$$
\zeta^{0}(t, x)=0 \quad \text { for a.e. }(t, x) \in\{(t, x): u(t, x)=\kappa\}
$$

for any fixed $\kappa \in \mathbb{R}$. Next, we have

- $f\left(u^{\varepsilon_{n}}\right) \rightarrow f(u)$ in $L_{l o c}^{1}(] 0, T / 2[\times \mathbb{R})$;

- up to a subsequence, $\operatorname{sgn}\left(u^{\varepsilon_{n}}-\kappa\right) \rightarrow \operatorname{sgn}(u-\kappa)$ a.e. in $\{(t, x): u(t, x) \neq \kappa\}$;

- there exists $w \in L^{\infty}(] 0, T / 2[\times \mathbb{R})$ such that, up to a subsequence,

$$
\operatorname{sgn}\left(u^{\varepsilon_{n}}-\kappa\right) \stackrel{\star}{\rightarrow} w \quad \text { in } L^{\infty}(] 0, T / 2[\times \mathbb{R}),
$$

and

$$
w(t, x)=\operatorname{sgn}(u(t, x)-\kappa) \quad \text { a.e. in }\{(t, x): u(t, x) \neq \kappa\} .
$$

It follows that, up to a subsequence, passing to the limit in (3.10) as $\varepsilon=\varepsilon_{n} \rightarrow 0$, we get that $u$ fulfills

$$
\int_{0}^{T / 2} \int_{\mathbb{R}}|u-\kappa| \partial_{t} \varphi+\operatorname{sgn}(u-\kappa)(f(u)-f(\kappa)) \partial_{x} \varphi \mathrm{d} x \mathrm{~d} t+\int_{0}^{T / 2} \int_{\mathbb{R}} \operatorname{sgn}(u-\kappa) \zeta^{0}(t, x) \varphi \mathrm{d} x \mathrm{~d} t \geq 0,
$$

for any non negative smooth function $\varphi$ with compact support, and hence is a weak entropy solution to (1.1) and (1.2).

Remark 3.2. Observe that, using (3.2), (3.3), (3.7) and (3.9) we have

$$
\zeta^{\varepsilon}(t, x)=-\frac{2}{T-2 t} u^{\varepsilon}(t, x)
$$

Hence, the control $z$ appearing in Lemma 3.1 can be written in feedback form as

$$
\zeta^{0}(t, x)=-\frac{2}{T-2 t} u(t, x)
$$

and $u=u(t, x)$ is the weak entropy solution solution of the singular balance law

$$
\partial_{t} u+\partial_{x} f(u)=-\frac{2}{T-2 t} u, \quad t \in[0, T / 2[, \quad x \in \mathbb{R},
$$

augmented with the initial datum $u(0, x)=u_{0}(x)$. Since the control $\zeta^{0}$ turns out to be uniformly bounded, we get that

$$
|u(t, x)| \leq C(T-2 t)
$$

for some positive constant $C$, and hence $u(t, \cdot)$ approaches uniformly zero as $t \rightarrow T / 2$.

\subsection{Step 2 - A locally Lipschitz continuous backward solution}

Now we proceed with the second step of the proof of Theorem 2.2.

Lemma 3.3. Let $v \in L^{1}(\mathbb{R}) \cap \mathrm{BV}_{\text {loc }}(\mathbb{R})$ satisfy (1.7). Then there exist $\tau_{v}>0$ and a weak entropy solution $w=w(t, x)$ to

$$
\partial_{t} w+\partial_{x} f(w)=0, \quad T-\tau_{v} \leq t<T,
$$

such that

- $w(t, \cdot)$ is locally Lipschitz continuous for any $t \in\left[T-\tau_{v}, T[\right.$;

- $w(t, \cdot) \rightarrow v$ in $L_{l o c}^{1}(\mathbb{R})$ as $t \rightarrow T$. 
Proof. In order to construct $w$, we follow an idea introduced in [5], and draw the candidate generalized backward characteristics [12]. In the following, being $v \in \mathrm{BV}_{l o c}(\mathbb{R})$, without loss of generality, we assume that $v$ is right continuous, i.e.

$$
v(\xi)=\lim _{x \rightarrow \xi^{+}} v(x) \quad \forall \xi \in \mathbb{R} .
$$

First of all, we observe that (1.7) implies that

$$
v(x)-v(y) \leq C(x-y), \quad \forall x>y,
$$

for some positive constant $C$. and hence the positive total variation of $v$ is bounded on bounded intervals. Let

$$
\xi(t, x)=x+(t-T) f^{\prime}(v(x)),
$$

so that $t \mapsto \xi(t, x)$ is a candidate backward characteristic originated at $(T, x)$. We claim that there exists $\left.\tau_{v} \in\right] 0, T\left[\right.$ such that $x \mapsto \xi(t, x)$ is increasing for any $t \in\left[T-\tau_{v}, T\right.$ [, i.e. there holds

$$
x_{1}<x_{2} \quad \Longrightarrow \xi\left(t, x_{1}\right)<\xi\left(t, x_{2}\right) \quad \forall \in\left[T-\tau_{v}, T[.\right.
$$

Indeed, if $v\left(x_{1}\right) \geq v\left(x_{2}\right)$, then $f^{\prime}\left(v\left(x_{1}\right)\right) \geq f^{\prime}\left(v\left(x_{2}\right)\right)$, being $f$ strictly convex, and hence

$$
\xi\left(t, x_{1}\right)-\xi\left(t, x_{2}\right)=x_{1}-x_{2}+(t-T)\left[f^{\prime}\left(v\left(x_{1}\right)\right)-f^{\prime}\left(v\left(x_{2}\right)\right)\right] \leq x_{1}-x_{2}<0 .
$$

Otherwise, let $L_{v}$ a Lipschitz constant for $f^{\prime}$ in the interval $\left[-\|v\|_{\infty},\|v\|_{\infty}\right]$. Then, using (3.12), we have

$$
\begin{aligned}
\xi\left(t, x_{1}\right)-\xi\left(t, x_{2}\right) & =x_{1}-x_{2}+(t-T)\left[f^{\prime}\left(v\left(x_{1}\right)\right)-f^{\prime}\left(v\left(x_{2}\right)\right)\right] \\
& \leq x_{1}-x_{2}+L_{v}(t-T)\left[v\left(x_{1}\right)-v\left(x_{2}\right)\right] \\
& \leq\left(x_{1}-x_{2}\right)\left[1+L_{v} C(t-T)\right] .
\end{aligned}
$$

If we choose $0<\tau_{v}<T-1 /\left(2 L_{v} C\right)$, we get that $\xi\left(t, x_{1}\right)<\xi\left(t, x_{2}\right)$ for all $t \in\left[T-\tau_{v}, T\right.$, as we claimed. Since $x \mapsto \xi(t, x)$ is increasing, it has at most countably many points of jump, say $x_{1}<x_{2}<\ldots<x_{n}<\ldots$, that, by construction, do not depend on $t$. We let

$$
\begin{gathered}
\xi^{-}\left(t, x_{n}\right)=x_{n}+(t-T) f^{\prime}\left(v\left(x_{n}-\right)\right), \\
E_{n}=\left\{(t, x) \in\left[T-\tau_{v}, T\right] \times \mathbb{R}: x \in\left[\xi^{-}\left(t, x_{n}\right), \xi\left(t, x_{n}\right)[\} .\right.\right.
\end{gathered}
$$

Now define

$$
w(t, x)=\left\{\begin{array}{ll}
v(y) & \text { if } \quad x=\xi(t, y) \exists y \in \mathbb{R}, \\
g\left(\frac{x-x_{n}}{t-T}\right) & \text { if } \quad(t, x) \in E_{n},
\end{array} \quad(t, x) \in\left[T-\tau_{v}, T\right] \times \mathbb{R},\right.
$$

where, as usual, $g=\left(f^{\prime}\right)^{-1}$. By construction $w \rightarrow v$ in $L_{l o c}^{1}(\mathbb{R})$ as $t \rightarrow T$ and it is constant along the characteristic lines of (3.11). Moreover, with the same arguments used in ([5], Proof of Thm. 1), it can be proved that $w(t, \cdot)$ is locally Lipschitz continuous for any $t \in\left[T-\tau_{v}, T\right.$. Hence, $w$ is a weak entropy solution to (3.11).

\subsection{Step 3 - Conclusion of the proof of Theorem 2.2}

Now we are ready to glue together the solutions and the controls constructed at Lemmas 3.1 and 3.3

Lemma 3.4. Let $0<t_{1}<t_{2}$ and $u_{1}, u_{2}$ be Lipschitz continuous functions. Then, there exists $\zeta^{1} \in$ $L^{\infty}(] 0, T[\times \mathbb{R})$ such that the weak entropy solution $u$ to

$$
\left\{\begin{array}{l}
\partial_{t} u+\partial_{x} f(u)=\zeta^{1}(t, x) \\
u\left(t_{1}, x\right)=u_{1}(x)
\end{array} \quad t \in\left[t_{1}, t_{2}\right],\right.
$$

is Lipschitz continuous and satisfies $u\left(t_{2}, x\right)=u_{2}(x)$ for all $x \in \mathbb{R}$. 


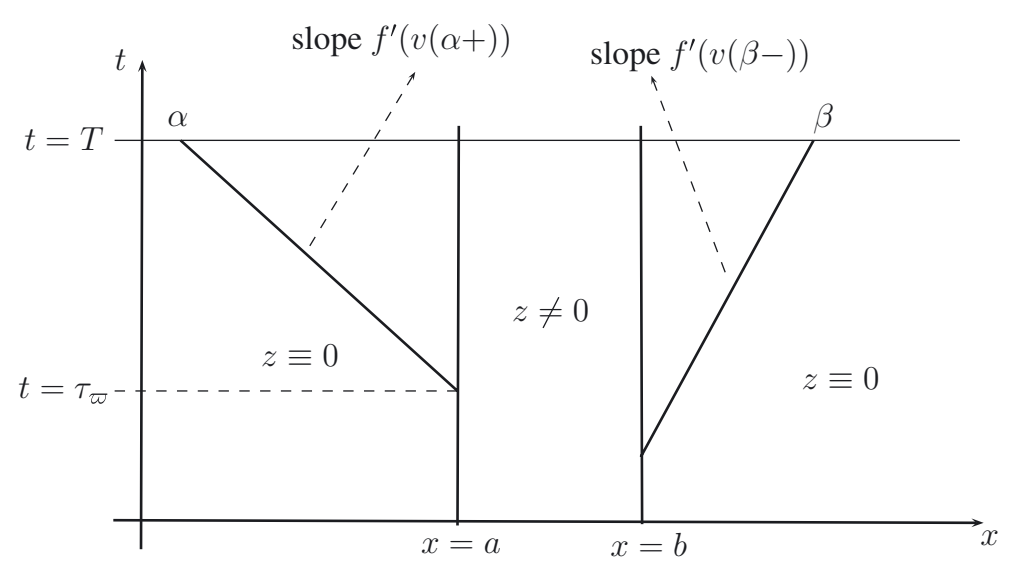

Figure 4. The domains of determinacy of $u$.

Proof. The proof is straightforward: let

$$
u(t, x)=u_{1}(x)+\left[u_{2}(x)-u_{1}(x)\right] \frac{t-t_{1}}{t_{2}-t_{1}}, \quad \zeta^{1}(t, x) \doteq \partial_{t} u(t, x)+\partial_{x} f(u(t, x)) .
$$

Then, $\zeta^{1} \in L^{\infty}(] 0, T[\times \mathbb{R})$ and $u$ fulfills the requirements.

Now, let $\left.\tau_{v} \in\right] 0, T / 2\left[\right.$ and $w$ be as in Lemma 3.3. Let $\zeta^{0}$ be as in Lemma 3.1 and $\zeta^{1}$ as in Lemma 3.4 with $t_{1}=T / 2, t_{2}=T-\tau_{v}, u_{1}(x) \equiv 0$ and $u_{2}(x)=w\left(T-\tau_{v}, x\right)$. Set

$$
z(t, x)= \begin{cases}\zeta^{0}(t, x) & \text { if } t \in[0, T / 2], \\ \zeta^{1}(t, x) & \text { if } \left.t \in] T / 2, T-\tau_{v}\right], \\ 0 & \text { if } \left.t \in] T-\tau_{v}, T\right] .\end{cases}
$$

Then, thanks to Lemmas 3.1-3.4, the solution $u$ to $(1.1)-(1.2)$ fulfills $u(T, x)=v(x)$ for a.e. $x \in \mathbb{R}$. This ends the proof of Theorem 2.2 .

\section{Proof of Theorem 2.4}

Observe that if $u$ is a weak entropy solution to (1.1) with $z$ supported in $[0, T] \times[a, b]$, then $u$ is a weak entropy solution to the conservation law (1.6) in $[0, T] \times(\mathbb{R} \backslash] a, b[)$. In particular, if $u(T, \cdot)=v$, with $v$ satisfying the assumptions of Theorem 2.4, the $(t, x)$-plane is divided in domains of determinacy of $u$ by the maximal and minimal backward characteristic from $(T, \alpha)$ and $(T, \beta)$, respectively, with $\alpha$ and $\beta$ defined at (2.9) (see Fig. 4). For instance, consider the strip $[0, T] \times]-\infty, a]$, and let $\varpi=v(\alpha+)$. To fix the ideas, assume $\alpha<a$, and trace the candidate maximal backward characteristic form $(T, \alpha)$, i.e. the line

$$
x=\alpha+f^{\prime}(\varpi)(t-T), \quad \varpi=v(\alpha+) .
$$

Let $\left.\tau_{\varpi} \in\right] 0, T\left[\right.$ be as at 2.16, so that $\tau_{\varpi}$ is the time at which the line (4.1) intersects the line $x=a$. Since $u_{0} \equiv 0$ and since we exert a control on $[0, T] \times[a, b]$, we can affect the behavior of a solution $u$ to (1.1) at the left of the line (4.1) for $x<a$ by controlling $u$ in the region $\left[0, \tau_{\varpi}\right] \times[a, b]$. Conversely, we can affect the behavior of $u$ at the right of (4.1) for $x<a$ by controlling its behavior in the region $\left[\tau_{\varpi}, T\right] \times[a, b]$.

Using the above hints, the proof of Theorem 2.4 is done in three steps too. 
1) In Section 4.1 we construct a control $z=z_{\chi}$ supported in the strip $[0, T] \times\left[a,+\infty\left[\right.\right.$ that drives $u_{0} \equiv 0$ to

$$
\chi(x)= \begin{cases}0 & \text { if } \quad x<\alpha, \\ \varpi & \text { if } \quad x \geq \alpha,\end{cases}
$$

where $\alpha \leq a$ and $\varpi<0$ are given. In this way we construct a solution $u_{\chi}$ to $(1.1)$, with $u_{\chi}(0, \cdot) \equiv 0$ and $u_{\chi}(T, \alpha+)=\varpi$.

2) In Section 4.2 we consider again controls supported in the strip $[0, T] \times[a,+\infty[$, but a more general final profile $v$ fulfilling conditions (1.7), (2.7), (2.10) and (2.12), with $\alpha$ defined at (2.9). We will use the results of Section 4.1 with $\varpi=v(\alpha+)$ in order to obtain a control $z$ for $t<\tau_{\varpi}$. Indeed, letting $u_{\chi}$ as above, the solution $u$ that we will obtain in Section 4.2 will satisfy $u(t, x)=u_{\chi}(t, x)$ whenever $t<\tau_{\varpi}$ or $(t, x)$ is at the left of the line (4.1). Then, we will use the theory of generalized characteristics by Dafermos [12] to construct a control $z$ and a solution $u$ for $\tau_{\varpi}<t<T$ and $(t, x)$ at the right of (4.1).

3) It is straightforward that the results obtained in Sections 4.1 and 4.2 can be extended to controls supported in the strip $[0, T] \times]-\infty, b]$. In the final step of the proof of Theorem 2.4 we suitably merge together the solutions and the controls obtained in the previous steps, and construct a bounded control $z$ supported in $[0, T] \times[a, b]$ driving $u_{0} \equiv 0$ to a final profile $v$ fulfilling the assumptions of the theorem.

We point out that we will construct in the meantime the control $z$ and the solution $u$ to (1.1) driving $u_{0} \equiv 0$ to $v$ in time $T$.

\subsection{Step 1 - Preliminary results}

We start by constructing a control driving $u_{0} \equiv 0$ to the function $\chi$ at $(4.2)$. Recall the definitions of $\mathcal{A}(T, \mathcal{Z})$ and $\mathcal{Z}_{a, b}, a, b \in \mathbb{R}$, at (1.4) and (1.5), respectively, and Lemmas 2.6 and 2.8 that will be used in the following.

Lemma 4.1. Let $\varpi<0$ and $\alpha \leq a$ be given with

$$
f^{\prime}(\varpi)<\frac{\alpha-a}{T}
$$

Then the function $\chi$ at $(4.2)$ belongs to $\mathcal{A}\left(T, \mathcal{Z}_{a,+\infty}\right)$. More precisely, we can find a measurable bounded function $z_{\chi}$ supported in $[0, T] \times\left[a,+\infty\left[\right.\right.$ such that the weak entropy solution $u=u_{\chi}$ to (1.1) and (1.2) with $u_{0} \equiv 0$ and $z=z_{\chi}$ satisfies $u_{\chi}(T, \cdot)=\chi$. Moreover, $z_{\chi}$ can be chosen so that $u_{\chi}$ is piecewise Lipschitz continuous suffering a discontinuity along a single Lipschitz continuous curve.

Proof. First of all, observe that it suffices to prove the lemma assuming $\alpha<a$. Indeed, if $\alpha=a$, we can choose $\delta>0$ such that

$$
f^{\prime}(\varpi)<\frac{\alpha-(a+\delta)}{T},
$$

and prove that $\chi \in \mathcal{A}\left(T, \mathcal{Z}_{a+\delta,+\infty}\right) \subseteq \mathcal{A}\left(T, \mathcal{Z}_{a,+\infty}\right)$. We recall the definition of $g=\left(f^{\prime}\right)^{-1}$ at (2.1). Moreover, we will let and $\tau_{\varpi}$ be defined as at (2.16) (see also Rem. 2.7). We distinguish four cases, depending on $f^{\prime}(0)$.

1) $f^{\prime}(0) \leq(\alpha-a) / T$.

We observe preliminarily that $[f(\varpi)-f(0)] / \varpi<(\alpha-a) / T$, being $\varpi<0$. We let

$$
\tau_{0}=T-\frac{\alpha-a}{f^{\prime}(0)},
$$

so that $\tau_{0}$ is the time at which the (candidate) minimal characteristics from $(T, \alpha)$ reaches $x=a$. We will construct a function $u$ containing a compression wave that generates a shock at $x=\alpha$ and $t=T$ with left 


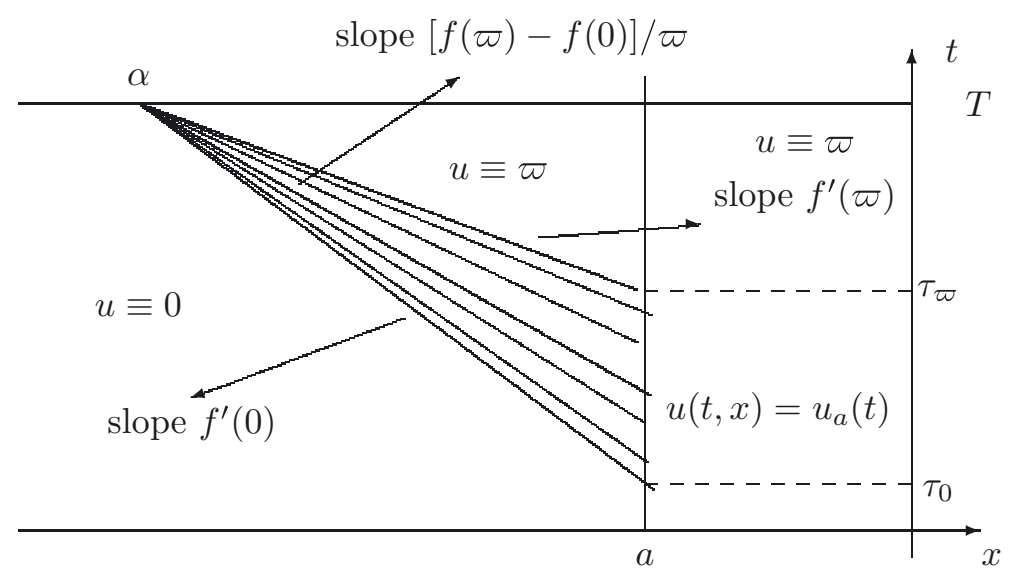

Figure 5. The case $[f(\varpi)-f(0)] / \varpi<(\alpha-a) / T$ and $f^{\prime}(0) \leq(\alpha-a) / T$.

and right states 0 and $\varpi$ respectively (see Fig. 5). Then, $z$ is defined in order to let $u$ satisfy a balance law. We set

$$
u_{a}(t)=\left\{\begin{array}{lll}
0 & \text { if } \quad t<\tau_{0}, \\
g\left(\frac{\alpha-a}{T-t}\right) & \text { if } \quad \tau_{0} \leq t<\tau_{\varpi}, \\
\varpi & \text { if } \quad t \geq \tau_{\varpi} .
\end{array}\right.
$$

Then we let

$$
u_{\chi}(t, x)=\left\{\begin{array}{lll}
0 & \text { if } \quad x<\alpha+f^{\prime}(0)(t-T) \text { and } x<a, \\
g\left(\frac{\alpha-x}{T-t}\right) & \text { if } \quad \alpha+f^{\prime}(0)(t-T) \leq x<\alpha+f^{\prime}(\varpi)(t-T) \text { and } x<a, \\
\varpi & \text { if } \quad \alpha+f^{\prime}(\varpi)(t-T) \leq x<a, \\
u_{a}(t) & \text { if } \quad x \geq a .
\end{array}\right.
$$

It is straightforward that $u=u_{\chi}(t, x)$ is locally Lipschitz continuous for $t<T$ and that $u_{\chi}(T, \cdot)=\chi$. Moreover, by construction $u$ fulfills (1.1) $-(1.2)$ with $u_{0} \equiv 0$, and $z=z_{\chi}$ where

$$
z_{\chi}(t, x)= \begin{cases}0 & \text { if } x<a \text { or } x \geq a \text { and } t \notin\left[\tau_{0}, \tau_{\varpi}[\right. \\ \partial_{t} u_{a}(t)=-\frac{1}{(T-t)^{2}} g^{\prime}\left(\frac{\alpha-a}{T-t}\right) & \text { if } x \geq a \text { and } \tau_{0} \leq t<\tau_{\varpi} .\end{cases}
$$

2) $(\alpha-a) / T<f^{\prime}(0)<0$ and $[f(\varpi)-f(0)] / \varpi<(\alpha-a) / T$.

In this case the characteristic from $(T, \alpha)$ with speed $f^{\prime}(0)$ does not reach $x=a$ in time $T$. We construct a control $z$ and a solution $u$ to (1.1) and (1.2) in a way similar to the previous case. We will use a compression wave that generates a shock issuing from the point of intersection in the $(t, x)$ plane between the characteristic from $(0, a)$ with speed $f^{\prime}(0)$ and the line

$$
x=\alpha+\frac{f(\varpi)-f(0)}{\varpi}(t-T),
$$




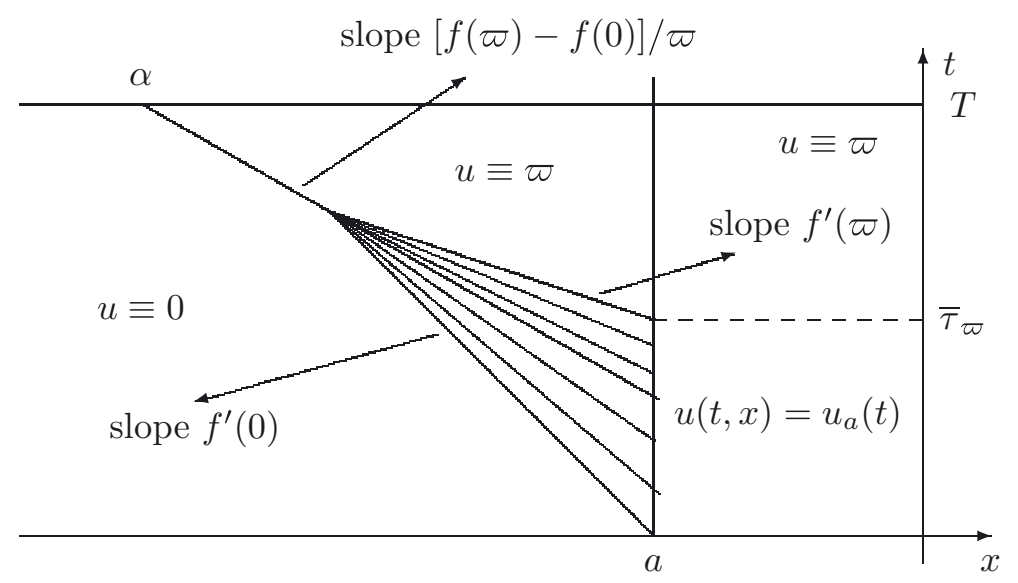

Figure 6 . The case $(\alpha-a) / T<f^{\prime}(0)<0$ and $[f(\varpi)-f(0)] / \varpi<(\alpha-a) / T$.

i.e. the point $(\tau, \xi)$ with

$$
\begin{aligned}
& \tau=\frac{\varpi}{\varpi f^{\prime}(0)-f(\varpi)+f(0)}\left[\alpha-a-T \frac{f(\varpi)-f(0)}{\varpi}\right], \\
& \xi=a+\frac{f^{\prime}(0) \varpi}{\varpi f^{\prime}(0)-f(\varpi)+f(0)}\left[\alpha-a-T \frac{f(\varpi)-f(0)}{\varpi}\right] .
\end{aligned}
$$

Since the construction is entirely similar to the previous one, we omit it and refer to Figure 6, where

$$
\bar{\tau}_{\varpi}=\tau-\frac{\xi-a}{f^{\prime}(\varpi)}
$$

is the time at which the characteristic from $(\tau, \xi)$ with speed $f^{\prime}(\varpi)$ reaches the line $x=a$.

3) $f^{\prime}(0)>0$.

Recall the definition of $\tau_{\varpi}$ at (2.16), and fix $\left.\tau \in\right] 0, \tau_{\varpi}[$. Let $\phi:[\tau, T] \rightarrow \mathbb{R}$ the function appearing in Lemma 2.6, and assume that $\phi$ is chosen according to Remark 2.7. Let $\eta_{1}:[0, \tau] \rightarrow\left[a,+\infty\left[\right.\right.$ be any $\mathcal{C}^{2}$, concave function such that (see Fig. 7).

$$
\eta_{1}(0)=\eta_{1}(\tau)=a, \quad \eta_{1}^{\prime}(0)=f^{\prime}(0), \quad \eta_{1}^{\prime}(\tau)=\frac{f(\phi(\tau))-f(0)}{\phi(\tau)},
$$

so that we have $\eta_{1}(t)>a$ for any $\left.t \in\right] 0, \tau[$. Since $f$ is strictly convex, the function

$$
]-\infty, 0] \ni v \mapsto F(v) \doteq \begin{cases}\frac{f(v)-f(0)}{v} & \text { if } v \neq 0, \\ f^{\prime}(0) & \text { if } v=0,\end{cases}
$$

is strictly increasing, and hence injective. Call $B$ its inverse, so that

$$
B=F^{-1} .
$$

Observe that, being $f \in \mathcal{C}^{2}(\mathbb{R}), F$ is a $\mathcal{C}^{1}$ function with $F^{\prime}(v)>0$ for any $v$, and that its inverse $B$ inherits the same regularity. For any $t \in[0, \tau]$ we set

$$
v(t)=B\left(\eta_{1}^{\prime}(t)\right)
$$




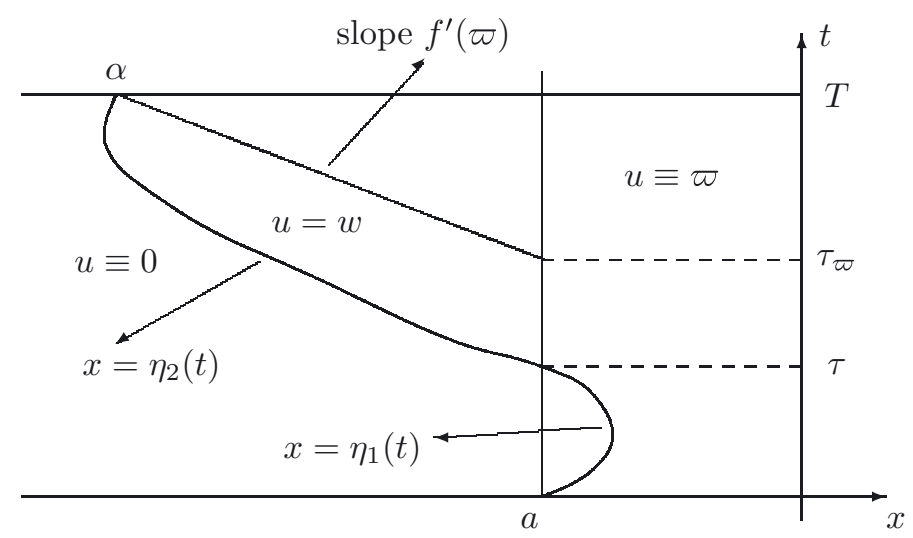

Figure 7 . The case $f^{\prime}(0)>0$.

so that $[0, \tau] \ni t \mapsto v(t)$ is a $\mathcal{C}^{1}$ function, due to the regularity properties of $B$ and $\eta_{1}$. Moreover, observe that by construction $v(t)<0$ whenever $t>0$, and $t \mapsto \eta_{1}(t)$ fulfills

$$
\eta_{1}^{\prime}(t)=\frac{f(v(t))-f(0)}{v(t)}
$$

so that $v(\tau)=\phi(\tau)$. Now, let $w=w(t, x)$ be the solution to the initial boundary value problem $(2.17)$, as it appears at Lemma 2.6. Call

$$
x=\eta_{2}(t), \quad t \in[\tau, T]
$$

the shock wave suffered by $w$ (see Fig. 7). Let

$$
\eta(t) \doteq \begin{cases}\eta_{1}(t) & \text { if } \quad t \in[0, \tau[ \\ \eta_{2}(t) & \text { if } \quad t \in[\tau, T]\end{cases}
$$

and set

$$
u_{\chi}(t, x) \doteq \begin{cases}w(t, x) & \text { if } x<a, \\ 0 & \text { if } a \leq x<\eta_{1}(t) \text { and } t \in[0, \tau[, \quad t \in[0, T] . \\ v(t) & \text { if } x \geq \eta_{1}(t) \text { and } t \in[0, \tau[, \\ \phi(t) & \text { if } x \geq a \text { and } t \in[\tau, T],\end{cases}
$$

Then, $u_{\chi}$ is piecewise Lipschitz continuous, suffering a shock discontinuity at $x=\eta(t)$, and $u_{\chi}(0, x) \equiv 0$. By construction the Rankine-Hugoniot conditions

$$
\eta^{\prime}(t)=\frac{f\left(u_{\chi}(t, \eta(t)+)-f\left(u_{\chi}(t, \eta(t)-)\right.\right.}{u_{\chi}(t, \eta(t)+)-u_{\chi}(t, \eta(t)-)}
$$

are satisfied, together with the entropy conditions (2.5) at $x=\eta(t)$. Moreover, $u$ fulfills a.e. the balance equation (1.1) with $z=z_{\chi}$ given by

$$
z_{\chi}(t, x) \doteq \begin{cases}0 & \text { if } x<a, \\ v^{\prime}(t) & \text { if } x \geq a \text { and } t \in[0, \tau[, \\ \phi^{\prime}(t) & \text { if } x \geq a \text { and } t \in[\tau, T] .\end{cases}
$$

Hence $u_{\chi}$ is an entropy admissible solution to (1.1) and (1.2) with $u_{0}(x) \equiv 0$. Being $u_{\chi}(T, x)=\chi(x)$ for any $x \in \mathbb{R}$, it follows that $z_{\chi}$ is a control we were looking for. 


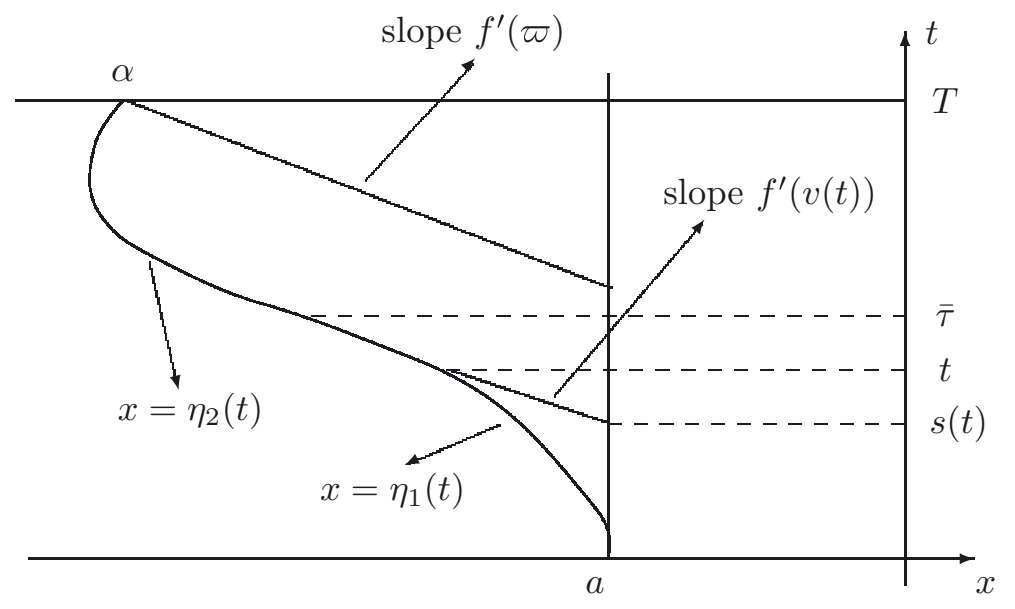

FiguRe 8 . The case $f^{\prime}(0)=0$.

4) $f^{\prime}(0)=0$.

The procedure is similar to the ones described in the previous cases, thus we omit the details, and refer to Figure 8. Let $\tau_{\varpi}$ be as at (2.16), and $0<\tau<\tau_{\varpi}, \phi, w$ and $\eta_{2}$ be as above. We construct a candidate shock curve $x=\eta(t)$,

$$
\eta(t) \doteq \begin{cases}\eta_{1}(t) & \text { if } \quad t \in[0, \bar{\tau}[, \\ \eta_{2}(t) & \text { if } \quad t \in[\bar{\tau}, T] .\end{cases}
$$

Here $\bar{\tau} \in] \tau, \tau_{\varpi}\left[\right.$ is chosen arbitrarily, and $t \mapsto \eta_{1}(t), t \in[0, \bar{\tau}]$ is a strictly concave function, such that

$$
\eta_{1}(0)=a, \quad \eta_{1}(\bar{\tau})=\eta_{2}(\bar{\tau}), \quad \eta_{1}^{\prime}(0)=0, \quad \eta_{1}^{\prime}(\bar{\tau})=\eta_{2}^{\prime}(\bar{\tau}) .
$$

Define $t \mapsto v(t), t \in[0, \bar{\tau}]$, as at (4.4)-(4.6), and observe that $v(\bar{\tau})=w(\bar{\tau}, \eta(\bar{\tau})+)$. It turns out that $v$ is a $\mathcal{C}^{1}$ function, and, due to the monotonicity property of the function $B$ at (4.4) and (4.5), it is strictly decreasing. Moreover, $v(0)=a$ and $(4.7)$ is satisfied for any $t \in[0, \bar{\tau}]$. This allows us to draw back the candidate maximal characteristics from $\left(t, \eta_{1}(t)\right)$, and to reconstruct the trace at $x=a$ of a candidate solution $u$ to an appropriate balance law. We let

$$
s(t)= \begin{cases}t+\frac{a-\eta_{1}(t)}{f^{\prime}(v(t))} & \text { if } \quad t \neq 0, \\ 0 & \text { if } \quad t=0,\end{cases}
$$

i.e. $s(t)$ is the time at which the candidate maximal backward characteristic from $\left(t, \eta_{1}(t)\right)$,

$$
x=\eta_{1}(t)+f^{\prime}(v(t))(s-t),
$$

intersects the line $x=a$. Such a function $s$ turns out to have a continuous inverse, which is defined on a time interval $[0, \tilde{\tau}]$, and which is continuously differentiable but at $t=0$. By letting $\psi(t)=v\left(s^{-1}(t)\right), t \in[0, \tilde{\tau}]$, one can prove that the control

$$
z_{\chi}(t, x)= \begin{cases}0 & \text { if } x<a, \\ \psi^{\prime}(t) & \text { if } x \geq a \text { and } t \in] 0, \tilde{\tau}[, \\ \phi^{\prime}(t) & \text { if } x \geq a \text { and } t \in[\tilde{\tau}, T],\end{cases}
$$

steers $u_{0}(x) \equiv 0$ to $\chi$ in time $T$.

The next lemma deals with the case $f^{\prime}(\varpi)=0$ and $\alpha=a$. 
Lemma 4.2. Let $\varpi<0$ be given with $f^{\prime}(\varpi)=0$. Then the function

$$
\widetilde{\chi}(x)= \begin{cases}0 & \text { if } \quad x<a, \\ \varpi & \text { if } \quad x \geq a,\end{cases}
$$

belongs to $\mathcal{A}\left(T, \mathcal{Z}_{a,+\infty}\right)$. More precisely, we can find a measurable bounded function $z_{\tilde{\chi}}$ supported in $[0, T] \times$ $\left[a,+\infty\left[\right.\right.$ such that the weak entropy solution $u=u_{\tilde{\chi}}$ to $(1.1)-(1.2)$ with $u_{0} \equiv 0$ and $z=z_{\tilde{\chi}}$ satisfies $u_{\tilde{\chi}}(T, \cdot)=\tilde{\chi}$. Moreover, $z_{\tilde{\chi}}$ can be chosen so that $u_{\tilde{\chi}}$ is piecewise Lipschitz continuous suffering a discontinuity along a single Lipschitz continuous curve.

Proof. Observe that, being $\varpi<0$ and $f^{\prime}$ strictly increasing, we have $f^{\prime}(0)>0$. We can proceed as in the proof of case 3) of Lemma 4.1, but we use Lemma 2.8, instead of Lemma 2.6. Since the proof is entirely similar, we omit it.

Remark 4.3. Since Lemma 2.8 and the construction in the proof of case 3 ) of Lemma 4.1, we can assume that the weak entropy solution $u_{\tilde{\chi}}$ of Lemma 4.2 satisfies

$$
u_{\widetilde{\chi}}(t, x)=\varpi \quad \forall t \in\left[\widetilde{\tau}_{\varpi}, T\right], x \geq a,
$$

with $\widetilde{\tau}_{\varpi}$ as in the statement of Lemma 2.8 .

In the following remark we summarize the construction carried out in the above Lemmas. This will be useful in Section 4.3 which contains the conclusion of the proof of Theorem 2.4.

Remark 4.4. As pointed out in the statement of Lemmas 4.1 and 4.2 , in order to attain the final profile $\chi$ at (4.2) we constructed a bounded control $z$ and a weak entropy solution $u$ to (1.1) which is piecewise Lipschitz continuous, suffering at most a shock discontinuity along a Lipschitz continuous curve $x=\eta(t)$. Along such a curve $u$ satisfies the Rankine-Hugoniot conditions (2.4) and the entropy conditions (2.5). Whenever $f^{\prime}(0) \geq 0$, it may happen that $\eta(t) \in[a, b]$ for $t \in[0, \tau]$, with $\tau \in] 0, T$ [ suitably chosen. In any case, by construction there exists a constant $C=C(\tau, \alpha, \varpi)>0$ depending on $\tau, \alpha$ and $\varpi$ such that

$$
\lim _{\tau \rightarrow 0} C(\tau, \alpha, \varpi)=0, \quad \max _{t \in[0, \tau]} \eta(t) \leq C(\tau, \alpha, \varpi) .
$$

Moreover, observe that the $L^{\infty}$ norm of the control $z$ satisfies

$$
\|z\|_{\infty}=\mathcal{O}(1) \cdot \frac{1}{\tau}
$$

where $\mathcal{O}(1)$ is the usual Landau's symbol. This is a key property of the control $z$ that we constructed, as it will be clear when completing the proof of Theorem 2.4 (see Rem. 4.8).

\subsection{Step 2 - Controls supported in a half-line}

In this section we consider controls supported in the strips $[0, T] \times[a,-\infty[$ and $[0, T] \times]-\infty, b]$, with $a, b \in \mathbb{R}$ given. We will give attainability results for general final profiles $v$, and the results of the previous subsection will play a key role.

Proposition 4.5. Let $u_{0} \equiv 0$ in (1.1) and (1.2), $v \in \mathrm{BV}(\mathbb{R})$, and $\alpha$ be defined as in (2.9). Assume that conditions (1.7), (2.7), (2.10) and (2.12) are fulfilled. Then $v \in \mathcal{A}\left(T, \mathcal{Z}_{a,+\infty}\right)$, and hence we can find a measurable bounded function $z=z_{a,+\infty}$ supported in $[0, T] \times\left[a,+\infty\left[\right.\right.$ such that the weak entropy solution $u=u_{a,+\infty}$ to $(1.1)$ and (1.2) with $u_{0} \equiv 0$ and $z=z_{a,+\infty}$ satisfies $u_{a,+\infty}(T, \cdot)=v$. Moreover, $z_{a,+\infty}$ can be chosen so that $u_{a,+\infty}$ is piecewise Lipschitz continuous suffering a discontinuity along a single Lipschitz continuous curve. 
Proof. Similarly to what we observed in Remark 2.5, assumptions (1.7) and (2.7) imply that there exists $\alpha \in \mathbb{R}$ such that $v(x) \neq 0$ only if $x \geq \alpha$. If $\alpha>a$, then, by using the same technique contained in the proof of Theorem 2.2, we obtain a bounded control $z$ driving $u \equiv 0$ to $v$ such that $z(t, x)=0$ for any $x \leq a$. Hence, without loss of generality, we can assume that $\alpha \leq a$. Moreover, it is not restrictive to assume that $v$ is right continuous. In order to prove the proposition we distinguish three cases, depending on $\alpha$ and

$$
\varpi \doteq v(\alpha+)=\lim _{x \rightarrow \alpha+} v(x) .
$$

We will mostly focus on the case $\varpi<0$ and $f^{\prime}(\varpi)<(\alpha-a) / T$, since the other ones can be treated in a similar way. Preliminarily, observe that the case $\varpi>0$ can not occur, otherwise

$$
\limsup _{h \rightarrow 0} \frac{v(\alpha+h)-v(\alpha)}{h}=+\infty
$$

and condition (1.7) would be violated.

1) $\varpi<0$ and $f^{\prime}(\varpi)<(\alpha-a) / T$.

First of all, as in the proof of Lemma 4.1, without loss of generality we can assume that $\alpha<a$. Otherwise we can choose $\delta>0$ such that

$$
f^{\prime}(\varpi)<\frac{\alpha-(a+\delta)}{T}
$$

and prove that $v \in \mathcal{A}\left(T, \mathcal{Z}_{a+\delta,+\infty}\right) \subseteq \mathcal{A}\left(T, \mathcal{Z}_{a,+\infty}\right)$. Let $\tau_{\varpi}$ and $\chi$ be defined as at (2.16) and (4.2), respectively, and let $\tau_{v}$ be as in Lemma 3.3. In order to construct the control $z$ we follow the same ideas of the proof of ([5], Thm. 1). We divide the time interval $[0, T]$ in three subintervals, $\left[0, \tau_{\varpi}\left[,\left[\tau_{\varpi}, T-\tau_{v}[\right.\right.\right.$, $\left[T-\tau_{v}, T\right]$. In $\left[0, \tau_{\varpi}\left[\right.\right.$ and $\left[T-\tau_{v}, T\right]$ we will perform the same constructions of Lemmas 4.1 and 3.3 , respectively. Next, we will let

$$
\xi(t, x)=x+(t-T) f^{\prime}(v(x))
$$

be the candidate backward characteristic from $(T, x)$. In the time interval $\left.] \tau_{\varpi}, T-\tau_{v}\right]$ we construct a Lipschitz continuous trace $u_{a}=u_{a}(t)$ of the candidate solution at $x=a$ by using the fact that the solution to (1.1) and (1.2) is constant along genuine characteristics for $x<a$. Finally, we will define a candidate Lipschitz solution $u$ in $] \tau_{\varpi}, T-\tau_{v}$ ] having $u_{a}$ as trace at $x=a$, and find a corresponding control $z$ such that $u$ is a solution to a system of balance laws. We will then obtain $z_{a,+\infty}$ and $u_{a,+\infty}$ by piecing together the contribution in suitable regions of the $(t, x)$-plane (see Fig. 9).

By Lemma 4.1 there exists a control $z_{\chi}$ driving $u_{0} \equiv 0$ to $\chi$ in time $T$. We let $z(t, x)=z_{\chi}(t, x)$ whenever $t \leq \tau_{\varpi}$ and $x \geq a$ and $u(t, x)=u_{\chi}(t, x)$ whenever

$$
\left.\left.t \in\left[0, \tau_{\varpi}\right] \quad \text { or } \quad t \in\right] \tau_{\varpi}, T\right] \text { and } \quad x<\alpha+f^{\prime}(\varpi)(t-T) .
$$

In this way we obtain a solution $u$ to (1.1) and (1.2) for $t \leq \tau_{\varpi}$ which is Lipschitz continuous in $\left[0, \tau_{\varpi}\right] \times \mathbb{R}$, but a Lipschitz curve where it suffers a jump discontinuity. Moreover, $u\left(\tau_{\varpi}, x\right)=\varpi$ for all $x \geq a$. In order to go further with the construction for $\left.t \in] \tau_{\varpi}, T\right]$, and particularly with the construction of the trace $u_{a}=u_{a}(t)$ at $x=a$ for $t \in] \tau_{\varpi}, T-\tau_{v}$ ], we proceed in three steps.

Step 1. First of all, observe that the function

$$
\left[\alpha, a\left[\ni x \mapsto \varphi(x) \doteq \frac{f^{\prime}(v(x))}{a-x}\right.\right.
$$

is strictly decreasing. Indeed, it suffices to prove that

$$
\limsup _{h \rightarrow 0} \frac{\varphi(x+h)-\varphi(x)}{h}<0 \quad \forall x \in[\alpha, a[.
$$


But this follows easily from (2.12), being

$$
\begin{aligned}
\limsup _{h \rightarrow 0} \frac{\varphi(x+h)-\varphi(x)}{h}= & \frac{f^{\prime \prime}(v(x))}{a-x}\left[\limsup _{h \rightarrow 0} \frac{v(x+h)-v(x)}{h}-\frac{f^{\prime}(v(x))}{f^{\prime \prime}(v(x))(x-a)}\right] \\
& \leq-\frac{c}{a-\alpha} \inf _{u \in \mathbb{R}} f^{\prime \prime}(u),
\end{aligned}
$$

where

$$
\sup \left\{\limsup _{h \rightarrow 0} \frac{v(x+h)-v(x)}{h}-\frac{f^{\prime}(v(x))}{(x-a) f^{\prime \prime}(v(x))}: x<a, v(x) \neq 0\right\}=-c<0 .
$$

From the monotonicity properties of $\varphi$ there follows that the lines (4.14) do not intersect each other in the strip $[0, T] \times]-\infty, a]$. Indeed, let $\alpha \leq x_{1}<x_{2}<a$ be such that

$$
x_{1}+(t-T) f^{\prime}\left(v\left(x_{1}\right)\right)=x_{2}+(t-T) f^{\prime}\left(v\left(x_{2}\right)\right) \doteq \xi .
$$

Being $\varphi\left(x_{1}\right)<\varphi\left(x_{2}\right)$, we have

$$
\frac{\xi-a}{x_{1}-a}=1+(T-t) \varphi\left(x_{1}\right)<1+(T-t) \varphi\left(x_{2}\right)=\frac{\xi-a}{x_{2}-a},
$$

from which we deduce that $\xi>a$.

Step 2. Due to the previous step, we deduce that $f^{\prime}(v(x))<0$ for all $x \in\left[\alpha, a\left[\right.\right.$. Indeed, if $f^{\prime}(v(\bar{x})) \geq 0$ for some $\bar{x} \in] \alpha, a\left[\right.$, being $f^{\prime}(\varpi) \leq(\alpha-a) / T<$ thanks to $(2.7)$, the lines

$$
x=\bar{x}+(t-T) f^{\prime}(v(\bar{x})), \quad x=\alpha+(t-T) f^{\prime}(\varpi)
$$

would intersect in the strip $[0, T] \times]-\infty, a]$. It follows that we can define a function

$$
[\alpha, a[\ni x \mapsto \tau(x), \quad \tau(x) \geq 0,
$$

such that $\xi(\tau(x), x)=$ a, i.e.

$$
\tau(x) \doteq T+\frac{a-x}{f^{\prime}(v(x))}=T+\frac{1}{\varphi(x)},
$$

that turns out to be strictly increasing, being $\varphi$ strictly decreasing. Hence, $x \mapsto \tau(x)$ has at most countably many points of discontinuity, say

$$
\alpha \leq x_{1}<x_{2}<\ldots<x_{n}<\ldots<a,
$$

where it has finite left and right limits, say $\tau\left(x_{k}-\right)$ and $\tau\left(x_{k}+\right)$, respectively, $k \geq 1$. Moreover, observe that, since (4.15) and recalling the definition of $\tau_{v}$ at Lemma 3.3, whenever $\tau(x) \leq T-\tau_{v}$ (and hence $\left.\tau_{v} \leq-1 / \varphi(x)\right)$ we have

$$
\tau\left(x_{2}\right)-\tau\left(x_{1}\right) \geq \gamma\left(x_{2}-x_{1}\right) \quad \forall x_{1}<x_{2} \in[\alpha, a[,
$$

where

$$
\gamma=\frac{\tau_{v}^{2} c}{a-\alpha} \inf _{u \in \mathbb{R}} f^{\prime \prime}(u)
$$

Step 3. Let $\tau^{-1}$ be the inverse function of $\tau(\cdot)$. Observe that $\tau^{-1}$ is strictly increasing and, moreover, since (4.17), there holds

$$
\left.\left.\tau^{-1}\left(t_{2}\right)-\tau^{-1}\left(t_{1}\right) \leq \frac{1}{\gamma}\left(t_{2}-t_{1}\right) \quad \forall t_{1}, t_{2} \in\right] \tau_{\varpi}, T-\tau_{v}\right], t_{1}<t_{2},
$$


and hence $\tau^{-1}$ is Lipschitz continuous on $] \tau_{\varpi}, T-\tau_{v}$ ]. In oder to produce a candidate Lipschitz trace $u_{a}=u_{a}(t)$ of $u=u(t, x)$ at $x=a$ in the time interval $\left.] \tau_{\varpi}, T-\tau_{v}\right]$, we let

$$
\bar{T} \doteq \sup _{x \in[\alpha, a[} \tau(x)
$$

and we distinguish two cases. We recall the notation $g=\left(f^{\prime}\right)^{-1}$.

(a) $\bar{T} \geq T-\tau_{v}$. For $\left.\left.t \in\right] \tau_{\varpi}, T-\tau_{v}\right]$ we define

$$
u_{a}(t) \doteq\left\{\begin{array}{lll}
v\left(\tau^{-1}(t)\right) & \text { if } \quad & \tau\left(x_{k-1}+\right)<t<\tau\left(x_{k}-\right) \text { for some } k \geq 1, \\
g\left(\frac{a-x_{k}}{t-T}\right) & \text { if } \quad & \tau\left(x_{k}-\right)<t<\tau\left(x_{k}+\right) \text { for some } k \geq 1 .
\end{array}\right.
$$

Such a map turns out to be Lipschitz continuous. Indeed, since (4.16), if $\tau\left(x_{k-1}+\right)<t<\tau\left(x_{k}-\right)$, we have

$$
v\left(\tau^{-1}(t)\right)=g\left(\frac{a-\tau^{-1}(t)}{t-T}\right) .
$$

Now the Lipschitz continuity of $t \mapsto u_{a}(t)$ follows easily, being $t-T$ bounded away from zero.

(b) $\bar{T}<T-\tau_{v}$. Observe that in this case there holds $f^{\prime}(v(a-))=0$. Indeed, we have

$$
f^{\prime}(v(a-))=\lim _{x \rightarrow a^{-}} f^{\prime}(v(x))=\lim _{x \rightarrow a-} \frac{a-x}{T-\tau(x)}=0,
$$

since

$$
\lim _{x \rightarrow a^{-}} \tau(x)=\bar{T}
$$

being $\tau(\cdot)$ strictly increasing. We define

$$
u_{a}(t) \doteq \begin{cases}v\left(\tau^{-1}(t)\right) & \text { if } \quad \tau\left(x_{k-1}+\right)<t<\tau\left(x_{k}-\right) \text { for some } k \geq 1, \\ g\left(\frac{a-x_{k}}{t-T}\right) & \text { if } \quad \tau\left(x_{k}-\right)<t<\tau\left(x_{k}+\right) \text { for some } k \geq 1 \\ v(a-) & \text { if } \quad \bar{T} \leq t \leq T-\tau_{v} .\end{cases}
$$

Again, $t \mapsto u_{a}(t)$ is Lipschitz continuous. Indeed, the Lipschitz continuity in $\left.] \tau_{\varpi}, \bar{T}\right]$ stems from what we have observed in the previous case. Hence, it suffices to prove that $u(\cdot)$ is continuous at $t=\bar{T}$. Since (4.19) and (4.20), it suffices to prove that

$$
\lim _{t \rightarrow \bar{T}} \tau^{-1}(t)=\sup _{t \in] \tau_{\varpi}, \bar{T}[} \tau^{-1}(t)=a .
$$

But this holds true thanks to (4.21).

Now, in order to construct a control $z$ and a solution $u$ to $(1.1)$ with $u(0, x) \equiv 0$ and $u(T, \cdot)=v$, we proceed as follows. The idea is to patch together suitable controls $z$ and the corresponding solutions to (1.1) (see Fig. 9).

(a) Following the same guidelines contained in the proof of Lemma 3.3, we can construct a solution $w=$ $w(t, x)$ to (3.11) in the time interval $\left[T-\tau_{v}, T\right]$ which is Lipschitz continuous in $\left[T-\tau_{v}, T[\times \mathbb{R}\right.$, and such that $w(t, \cdot) \rightarrow v$ in $L_{l o c}^{1}(\mathbb{R})$ as $t \rightarrow T$. Observe that by construction we have

$$
w\left(T-\tau_{v}, a\right)=u_{a}\left(T-\tau_{v}\right) .
$$




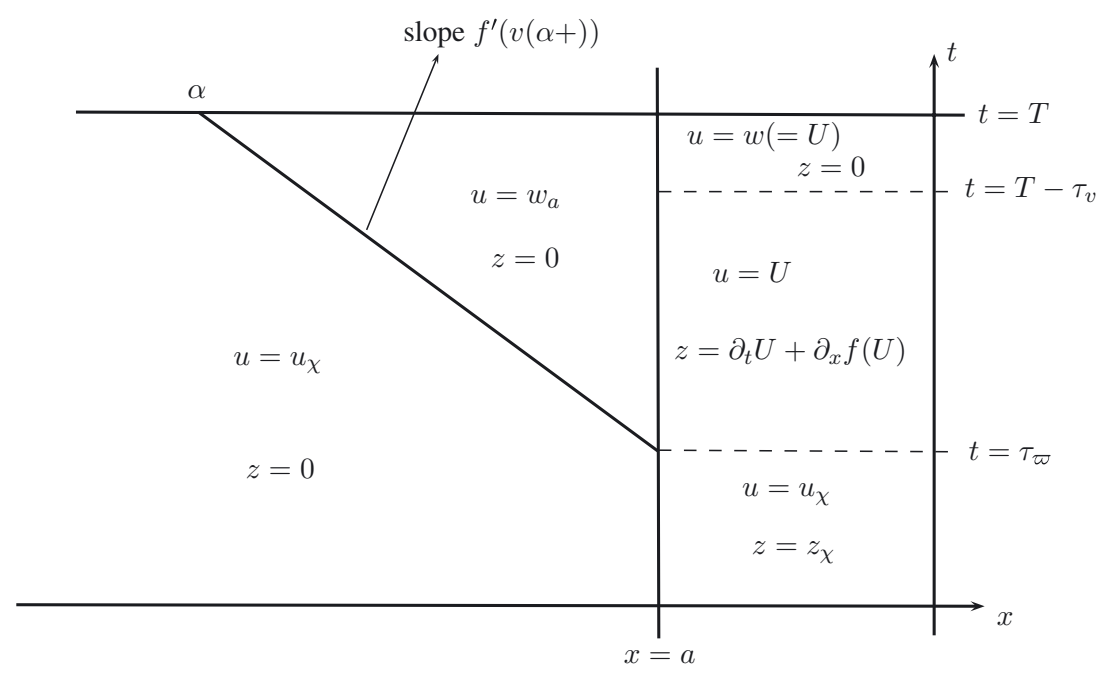

Figure 9. The construction of $z$ and $u$.

(b) Consider the strip $S \doteq\left[\tau_{\varpi}, T-\tau_{v}\right] \times[a,+\infty[$, and the function $U=U(t, x)$ defined on $\partial S$ by

$$
U(t, x)= \begin{cases}\varpi & \text { if } \quad t=\tau_{\varpi} \text { and } x \geq a, \\ w(t, x) & \text { if } t \in\left[T-\tau_{v}, T\right] \text { and } x \geq a, \\ u_{a}(t) & \text { if } t \in] \tau_{\varpi}, T-\tau_{v}[\text { and } x=a .\end{cases}
$$

By construction $U$ is Lipschitz continuous, and hence, using McShane-Whitney extension lemma (e.g., see [2], Prop. 2.12), it can be extended to a Lipschitz function, that we call again $U=U(t, x)$ defined in the all strip $S$.

(c) We let $w_{a}=w_{a}(t, x)$ the solution to

$$
\left\{\begin{array}{ll}
\partial_{t} u+\partial_{x} f(u)=0 & \\
u(t, a)=u_{a}(t) & \text { if } t \in\left[\tau_{\varpi}, T-\tau_{v}[,\right. \\
u(t, a)=w(t, a)) & \text { if } \left.t \in] T-\tau_{v}, T\right], \\
\varpi & \text { if } t=\tau_{\varpi},
\end{array} \quad t \in\left[\tau_{\varpi}, T\right], x \leq a .\right.
$$

Observe that, by construction,

$$
w_{a}(t, x)=w(t, x) \quad \forall t \in\left[T-\tau_{v}, T\right], \alpha+f^{\prime}(\varpi)(t-T)<x \leq a .
$$

(d) Recalling the definition of $u_{\chi}$ and $z_{\chi}$ at Lemma 4.1, we let (see Fig. 9)

$$
z_{a,+\infty}(t, x)= \begin{cases}z_{\chi}(t, x) & \text { if } t \in\left[0, \tau_{\varpi}\right], \\ \partial_{t} U(t, x)+\partial_{x} f(U(t, x)) & \text { if } t \in] \tau_{\varpi}, T-\tau_{v}[\text { and } x \geq a, \\ 0 & \text { otherwise, }\end{cases}
$$

and

$$
u_{a,+\infty}(t, x)= \begin{cases}u_{\chi}(t, x) & \text { if } t \in\left[0, \tau_{\varpi}\right] \text { or } x \leq \alpha+f^{\prime}(\varpi)(t-T), \\ U(t, x) & \text { if } \left.t \in] \tau_{\varpi}, T\right] \text { and } x \geq a \\ w_{a}(t, x) & \text { if } \left.t \in] \tau_{\varpi}, T\right] \text { and } \alpha+f^{\prime}(\varpi)(t-T)<x<a .\end{cases}
$$


By construction, it turns out that $u_{a,+\infty}$ solves (1.1) and (1.2) with $u_{0}(x) \equiv 0$ and $z=z_{a,+\infty}$, it fulfills $u(T, \cdot)=v$ and $z_{a,+\infty}$ is supported in the half line $[a,+\infty[$.

2) $\varpi<0, \alpha=a$ and $f^{\prime}(\varpi)=0$.

Let $\tau$ and $\widetilde{\tau}_{\varpi}$ be as in the statement of Lemma 2.8, and $z_{\tilde{\chi}}$ and $u_{\tilde{\chi}}$ be as at Lemma 4.2. As above, we let $\tau_{v}$ and $w=w(t, x)$ be as at Lemma 3.3. Moreover, let $x=\eta(t)$ the Lipschitz curve along which $u_{\tilde{\chi}}$ suffers a shock discontinuity, so that $\eta(0)=\eta(\tau)=a$ by construction. Using the McShane-Whitney extension lemma we call

$$
U=U(t, x), \quad t \in[\tau, T], x \geq a,
$$

a Lipschitz continuous extension of the function

$$
(t, x) \mapsto \begin{cases}u_{\tilde{\chi}}(\tau, x) & \text { if } \quad t=\tau \text { and } x>a, \\ u_{\tilde{\chi}}(t, a) & \text { if } t \in\left[\tau, \max \left\{T-\tau_{v}, \widetilde{\tau}_{\varpi}\right\}, T[\text { and } x=a,\right. \\ w(t, x) & \text { if } t \in\left[\max \left\{T-\tau_{v}, \widetilde{\tau}_{\varpi}\right\}, T\right] \text { and } x \geq a .\end{cases}
$$

Then, we let

$$
z_{a,+\infty}(t, x)= \begin{cases}z_{\tilde{\chi}}(t, x) & \text { if } t \in[0, \tau] \\ \partial_{t} U(t, x)+\partial_{x} f(U(t, x)) & \text { if } \left.t \in] \tau, \max \left\{T-\tau_{v}, \widetilde{\tau}_{\varpi}\right\}\right] \text { and } x \geq a \\ 0 & \text { otherwise }\end{cases}
$$

and

$$
u_{a,+\infty}(t, x)= \begin{cases}u_{\tilde{\chi}}(t, x) & \text { if } t \in[0, \tau] \text { or } x \leq a \\ U(t, x) & \text { if } \left.t \in] \tau, \max \left\{T-\tau_{v}, \widetilde{\tau}_{\varpi}\right\}\right] \text { and } x \geq a \\ w(t, x) & \text { if } \left.t \in] \max \left\{T-\tau_{v}, \widetilde{\tau}_{\varpi}\right\}, T\right] \text { and } x \geq a .\end{cases}
$$

It turns out that by construction $u=u_{a,+\infty}$ and $z=z_{a,+\infty}$ fulfill the desired requests.

3) $\varpi=0$.

Observe that thanks to $(2.7)$ there holds

$$
f^{\prime}(\varpi) \leq \frac{\alpha-a}{T-t}
$$

Two cases may happen.

(a) $\alpha<a$.

Let $\tau_{\varpi}$ be defined as at (2.16), so that $\tau_{\varpi} \in\left[0, T\left[\right.\right.$. We let $u(t)=u(t, a)=0$ for $0 \leq t \leq \tau_{\varpi}$, and then we proceed as in case $\varpi<0$ for constructing $u(t)$ for $\tau_{\varpi}<t \leq T-\tau_{v}$.

(b) $\alpha \geq a$.

We let $w=w(t, x)$ be as at Lemma 3.3, and call $U=U(t, x)$ a Lipschitz continuous function such that $U(t, x)=0$ for $x<\alpha$ or $t=0$ and $U\left(T-\tau_{v}, x\right)=w(t, x)$ for $x \geq \alpha$. Then, we proceed as at cases $\left.\mathbf{1}\right)$ and 3 ) to produce the control $z$ and the solution $u$ we were looking for.

Remark 4.6. Observe that condition (2.12) ensures that the profile of the candidate solution that we constructed at $x=a$ is Lipschitz continuous, and hence the control $z$ be $L^{\infty}$. Indeed, consider Burgers's equation

$$
\partial_{t} u+\partial_{x}\left(u^{2} / 2\right)=0
$$

and let $\tau=\tau(x) \in[1,3 / 2[, x \in[-1,0[$, be the solution to

$$
-\sqrt{3-2 \tau}(2-\tau)=x .
$$


Then it can be easily seen that

$$
v(x)= \begin{cases}0 & \text { if } \quad x<-1 \text { or } x>0, \\ -\sqrt{3-2 \tau(x)} & \text { if } \quad-1 \leq x<0,\end{cases}
$$

fulfills conditions (1.7), (2.7) with $a=0$ and $T=2$. Moreover, it satisfies

$$
\limsup _{h \rightarrow 0} \frac{v(x+h)-v(x)}{h}<\frac{f^{\prime}(v(x))}{x f^{\prime \prime}(v(x))}
$$

for $x \in[-1,0[$. Indeed, from (4.22) we get

$$
\tau^{\prime}(x)=\frac{\sqrt{3-2 \tau(x)}}{5-3 \tau(x)}
$$

so that for $x \in[-1,0[$ we obtain

$$
\limsup _{h \rightarrow 0} \frac{v(x+h)-v(x)}{h}=\frac{1}{5-3 \tau(x)} .
$$

In order that (4.23) be fulfilled, we must check that

$$
\frac{1}{5-3 \tau(x)}<-\frac{\sqrt{3-2 \tau(x)}}{x},
$$

and this is true thanks to $(4.22)$ and being $\tau(x)<3 / 2$. Nevertheless, using the construction contained in the proof of Proposition 4.5, we get that the candidate solution $u=u(t, x)$ at $x=0$ and for $t \in[1,3 / 2$ [ satisfies

$$
u(t, 0)=-\sqrt{3-2 t},
$$

so that we do not get a Lipschitz profile. Hence, we do not obtain an $L^{\infty}$ control $z(t, x)=\partial_{t} u(t, 0), x \geq 0$, supported in $[0,2] \times\left[0,+\infty\left[\right.\right.$ and steering to $v$ the solution $u$ to (1.1) and (1.2) with $f(u)=u^{2} / 2$ and $u_{0}=0$.

A statement which is symmetric to Proposition 4.5 can be proved.

Proposition 4.7. Let $u_{0} \equiv 0$ in (1.1) and (1.2), $v \in \mathrm{BV}(\mathbb{R})$, and $\beta$ be defined as in (2.9). Assume that conditions (1.7), (2.8), (2.11) and (2.13) are fulfilled. Then $v \in \mathcal{A}\left(T, \mathcal{Z}_{-\infty, b}\right)$, and hence we can find a measurable bounded function $z=z_{-\infty, b}$ supported in $\left.\left.[0, T] \times\right]-\infty, b\right]$ such that the weak entropy solution $u=u_{-\infty, b}$ to $(1.1)$ and (1.2) with $u_{0} \equiv 0$ and $z=z_{-\infty, b}$ satisfies $u_{-\infty, b}(T, \cdot)=v$. Moreover, $z_{-\infty, b}$ can be chosen so that $u_{-\infty, b}$ is piecewise Lipschitz continuous suffering a discontinuity along a single Lipschitz continuous curve.

It is obvious that the proof is entirely similar to the one of Proposition 4.5, and thus we omit it.

\subsection{Step 3 - Conclusion of the proof of Theorem 2.4}

We will obtain the control $z$ and the solution $u$ by patching together the controls and the solutions at Propositions 4.5 and 4.7 . To fix the ideas, let $f^{\prime}(0) \geq 0$. We will deal only with the case

$$
v(\alpha+)<0<v(\beta-), \quad \alpha<a, \quad \beta>b,
$$

the other ones being similar. Let $z_{a,+\infty}, u_{a,+\infty}$ and $z_{-\infty, b}$ and $u_{-\infty, b}$ be as in Propositions 4.5 and 4.7 , respectively. Then, $u_{a,+\infty}$ is Lipschitz continuous out of a Lipschitz's curve $x=\eta(t)$ fulfilling (4.11), where

$$
\eta(0)=\eta(\tau)=a \quad \text { and } \quad \eta(t) \geq a \quad \forall t \in[0, \tau]
$$




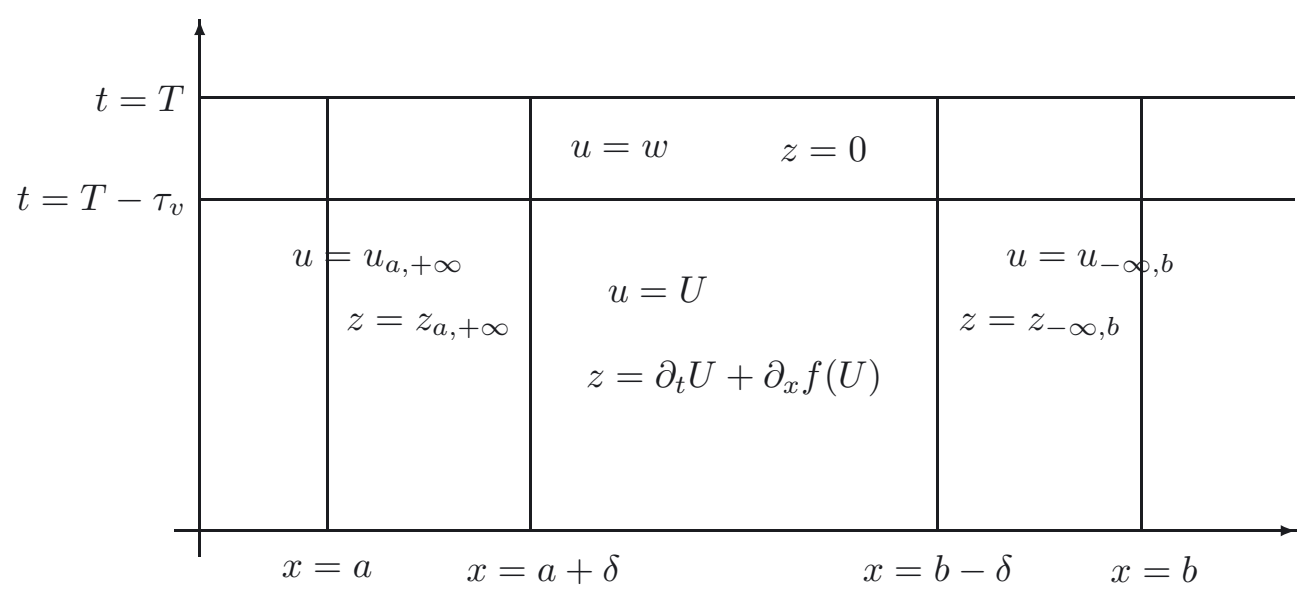

FiguRE 10. Conclusion of the proof of Theorem 2.4.

for a given $\tau \in] 0, T$. Hence, since Remark 4.4 we can choose $\tau$ such that

$$
\max _{t \in[0, \tau]} \eta(t)<b .
$$

Let $\delta>0$ be such that

$$
b-\delta>\max _{t \in[0, \tau]} \eta(t)+\delta .
$$

As usual, we let $\tau_{v}$ and $w$ be as in Lemma 3.3. Using again the McShane-Whitney extension lemma, call $U=U(t, x)$ the Lipschitz continuous extension to the whole rectangle

$$
\left[0, T-\tau_{v}\right] \times[a+\delta, b-\delta]
$$

of the function defined by

$$
(t, x) \mapsto \begin{cases}0 & \text { if } t=0 \text { and } x \in[a+\delta, b-\delta], \\ u_{a,+\infty}(t, a+\delta) & \text { if } t \in] 0, T-\tau_{v}[\text { and } x=a+\delta \\ u_{-\infty, b}(t, b-\delta) & \text { if } t \in] 0, T-\tau_{v}[\text { and } x=b-\delta \\ w\left(T-\tau_{v}, x\right) & \text { if, } t=T-\tau_{v} \text { and } x \in[a+\delta, b-\delta] .\end{cases}
$$

Then, let (see Fig. 10)

$$
z(t, x)= \begin{cases}z_{a,+\infty}(t, x) & \text { if } x \leq a+\delta \\ \partial_{t} U(t, x)+\partial_{x} f(U(t, x)) & \text { if } t \in\left[0, T-\tau_{v}\right] \text { and } x \in[a+\delta, b-\delta] \\ z_{-\infty, b}(t, x) & \text { if } x \geq b-\delta \\ 0 & \text { otherwise, }\end{cases}
$$

and

$$
u(t, x)= \begin{cases}u_{a,+\infty}(t, x) & \text { if } \quad x \leq a+\delta \\ U(t, x) & \text { if } t \in\left[0, T-\tau_{v}\right] \text { and } x \in[a+\delta, b-\delta], \\ w(t, x) & \text { if } \left.t \in] T-\tau_{v}, T\right] \text { and } x \in[a+\delta, b-\delta] \\ u_{-\infty, b}(t, x) & \text { if } x \geq b-\delta .\end{cases}
$$


It turns out that by construction $z$ is supported in $[0, T] \times[a, b], u$ is a weak entropy solution to (1.1) and (1.2) with $u_{0}(x) \equiv 0$, and $u(t, \cdot)=v$.

Remark 4.8. In view of Remark 4.4 and of the above construction, we point out that (4.11) and (4.12) are key properties in order to construct a bounded control $z$ steering $u_{0}=0$ to $v$ in time $T$, independently on the amplitude $b-a>0$ of the interval $[a, b]$. Conversely, using the above construction we obtain a control $z=z_{a, b}$ such that

$$
\left\|z_{a, b}\right\|_{\infty} \rightarrow+\infty \quad \text { as } \quad(b-a) \rightarrow 0
$$

\section{Appendix A.}

In this section we state and prove some technical lemmas that were used in Section 3. In the following, if $B \subset \mathbb{R}^{n}$ is a measurable set, we denote by $|B|$ its Lebesgue's measure. Moreover, we let $B_{p}=\pi^{(p) / 2} / \Gamma(n / 2+1)$ be the Lebesgue's measure of the unit ball in $\mathbb{R}^{p}$.

Lemma A.1. Let $\mathbf{k} \in \mathbb{R}^{n}, \mathbf{k} \neq \mathbf{0}$, and $r>0$. Then, if

$$
C_{\mathbf{k}} \doteq\left\{\mathbf{x} \in B_{r}(\mathbf{0}):|\langle\mathbf{k}, \mathbf{x}\rangle| \geq\|\mathbf{k}\|\|\mathbf{x}\| / 2\right\}
$$

there holds

$$
\left|C_{\mathbf{k}}\right|=r^{n}\left[B_{n-1} \frac{3^{(n-1) / 2}}{n 2^{n-1}}+\int_{1 / 2}^{1}\left(1-t^{2}\right)^{(n-1) / 2} \mathrm{~d} t\right]
$$

Proof. It is not restrictive to assume that $\mathbf{k}=k \mathbf{e}_{1}$, where $\left\{\mathbf{e}_{1}, \ldots, \mathbf{e}_{n}\right\}$ is the canonical basis of $\mathbb{R}^{n}$. Then

$$
C_{\mathbf{k}}=\left\{\left(x_{1}, \ldots, x_{n}\right) \in \mathbb{R}^{n}: x_{2}^{2}+\ldots+x_{n}^{2} \leq \min \left\{3 x_{1}^{2}, r^{2}-x_{1}^{2}\right\}\right\} .
$$

There follows that

$$
\begin{aligned}
\left|C_{\mathbf{k}}\right| & =\int_{-r}^{r} B_{n-1}\left|\min \left\{3 x^{2}, r^{2}-x^{2}\right\}\right|^{(n-1) / 2} \mathrm{~d} x \\
& =2 B_{n-1}\left[3^{(n-1) / 2} \int_{0}^{r / 2} x^{n-1} \mathrm{~d} x+\int_{r / 2}^{r}\left(r^{2}-x^{2}\right)^{(n-1) / 2} \mathrm{~d} x\right],
\end{aligned}
$$

from which the conclusion easily follows.

Now, we recall the following definition (see [2], Def. 3.70).

Definition A.2. Let $u \in L_{l o c}^{1}\left(\mathbb{R}^{n}\right)$. We say that $u$ is approximatively differentiable at $\mathbf{x}_{0} \in \mathbb{R}^{n}$ if there exist $\mathbf{L} \in \mathbb{R}^{n}$ such that

$$
\lim _{r \rightarrow 0} \frac{1}{\left|B_{r}\left(\mathbf{x}_{0}\right)\right|} \int_{B_{r}\left(\mathbf{x}_{0}\right)} \frac{\left|u(\mathbf{x})-u\left(\mathbf{x}_{0}\right)-\left\langle\mathbf{L}, \mathbf{x}-\mathbf{x}_{0}\right\rangle\right|}{\left\|\mathbf{x}-\mathbf{x}_{0}\right\|} \mathrm{d} \mathbf{x}=0
$$

where $\langle\cdot, \cdot\rangle$ denotes the usual scalar product in $\mathbb{R}^{n}$. In such a case, we write $\mathbf{L}=\nabla u\left(\mathbf{x}_{0}\right)$.

By a theorem due to Calderón and Zygmund (e.g., see [2], Thm. 3.83), if $\Omega \subseteq \mathbb{R}^{n}$ is open and $u \in \operatorname{BV}_{l o c}(\Omega)$, then $u$ is almost everywhere approximately differentiable.

Proposition A.3. Let $\Omega \subseteq \mathbb{R}^{n}$ be open, and $u \in \mathrm{BV}_{\text {loc }}(\Omega)$. Let $\nabla u(\mathbf{x})$ be the approximate differential of $u$ at $\mathbf{x}$. If $u$ is constant on a measurable set $A \subseteq \Omega$, then $\nabla u(\mathbf{x})=0$ for a.e. $\mathbf{x} \in A$. 
Proof. Let $|A|>0$, otherwise nothing has to be proved, and assume that $u(\mathbf{x})=\kappa$ for all $\mathbf{x} \in A$. Let $\mathbf{x}_{0} \in A$ be a point of approximate differentiability of $u$ such that

$$
\lim _{r \rightarrow 0} \frac{\left|B_{r}\left(\mathbf{x}_{0}\right) \cap A\right|}{\left|B_{r}\left(\mathbf{x}_{0}\right)\right|}=1,
$$

which holds for a.e. $\mathbf{x}_{0} \in A$. Then we have

$$
\begin{aligned}
0= & \lim _{r \rightarrow 0} \frac{1}{\left|B_{r}\left(\mathbf{x}_{0}\right)\right|} \int_{B_{r}\left(\mathbf{x}_{0}\right)} \frac{\left|u(\mathbf{x})-u\left(\mathbf{x}_{0}\right)-\left\langle\nabla u\left(\mathbf{x}_{0}\right), \mathbf{x}-\mathbf{x}_{0}\right\rangle\right|}{\left\|\mathbf{x}-\mathbf{x}_{0}\right\|} \mathrm{d} \mathbf{x} \\
= & \lim _{r \rightarrow 0} \frac{1}{\left|B_{r}\left(\mathbf{x}_{0}\right)\right|} \int_{B_{r}\left(\mathbf{x}_{0}\right) \cap A} \frac{\left|\left\langle\nabla u\left(\mathbf{x}_{0}\right), \mathbf{x}-\mathbf{x}_{0}\right\rangle\right|}{\left\|\mathbf{x}-\mathbf{x}_{0}\right\|} \mathrm{d} \mathbf{x} \\
& +\frac{1}{\left|B_{r}\left(\mathbf{x}_{0}\right)\right|} \int_{B_{r}\left(\mathbf{x}_{0}\right) \backslash A} \frac{\left|u(\mathbf{x})-u\left(\mathbf{x}_{0}\right)-\left\langle\nabla u\left(\mathbf{x}_{0}\right), \mathbf{x}-\mathbf{x}_{0}\right\rangle\right|}{\left\|\mathbf{x}-\mathbf{x}_{0}\right\|} \mathrm{d} \mathbf{x},
\end{aligned}
$$

and hence there holds

$$
\lim _{r \rightarrow 0} \frac{1}{\left|B_{r}\left(\mathbf{x}_{0}\right)\right|} \int_{B_{r}\left(\mathbf{x}_{0}\right) \cap A} \frac{\left|\left\langle\nabla u\left(\mathbf{x}_{0}\right), \mathbf{x}-\mathbf{x}_{0}\right\rangle\right|}{\left\|\mathbf{x}-\mathbf{x}_{0}\right\|} \mathrm{d} \mathbf{x}=0 .
$$

Now, assume by contradiction that $\nabla u\left(\mathbf{x}_{0}\right)=\mathbf{k} \neq \mathbf{0}$, and let

$$
C_{\mathbf{k}}=\left\{\mathbf{x} \in \mathbb{R}^{n}:\left|\left\langle\mathbf{k}, \mathbf{x}-\mathbf{x}_{0}\right\rangle\right| \geq\|\mathbf{k}\|\left\|\mathbf{x}-\mathbf{x}_{0}\right\| / 2\right\} .
$$

Then, due to lemma A.1, we have

$$
\lim _{r \rightarrow 0} \frac{\left|B_{r}\left(\mathbf{x}_{0}\right) \cap C_{\mathbf{k}}\right|}{\left|B_{r}\left(\mathbf{x}_{0}\right)\right|}=\frac{1}{B_{n}}\left[B_{n-1} \frac{3^{(n-1) / 2}}{n 2^{n-1}}+\int_{1 / 2}^{1}\left(1-t^{2}\right)^{(n-1) / 2} \mathrm{~d} t\right] \doteq \gamma>0 .
$$

Since (A.1), there holds

$$
\lim _{r \rightarrow 0} \frac{\left|B_{r}\left(\mathbf{x}_{0}\right) \backslash A\right|}{\left|B_{r}\left(\mathbf{x}_{0}\right)\right|}=0
$$

and hence

$$
\lim _{r \rightarrow 0} \frac{\left|\left(B_{r}\left(\mathbf{x}_{0}\right) \cap C_{\mathbf{k}}\right) \backslash A\right|}{\left|B_{r}\left(\mathbf{x}_{0}\right)\right|}=0
$$

so that

$$
\frac{\left|B_{r}\left(\mathbf{x}_{0}\right) \cap A \cap C_{\mathbf{k}}\right|}{\left|B_{r}\left(\mathbf{x}_{0}\right)\right|} \geq \frac{\gamma}{2}
$$

whenever $r>0$ is sufficiently small. But this implies that for the same values of $r>0$ there holds

$$
\begin{aligned}
& \frac{1}{\left|B_{r}\left(\mathbf{x}_{0}\right)\right|} \int_{B_{r}\left(\mathbf{x}_{0}\right) \cap A} \frac{\left|\left\langle\nabla u\left(\mathbf{x}_{0}\right), \mathbf{x}-\mathbf{x}_{0}\right\rangle\right|}{\left\|\mathbf{x}-\mathbf{x}_{0}\right\|} \mathrm{d} \mathbf{x} \geq \frac{1}{\left|B_{r}\left(\mathbf{x}_{0}\right)\right|} \int_{B_{r}\left(\mathbf{x}_{0}\right) \cap A \cap C_{\mathbf{k}}} \frac{\left|\left\langle\mathbf{k}, \mathbf{x}-\mathbf{x}_{0}\right\rangle\right|}{\left\|\mathbf{x}-\mathbf{x}_{0}\right\|} \mathrm{d} \mathbf{x} \\
& \geq \frac{\|\mathbf{k}\|}{2} \frac{\left|B_{r}\left(\mathbf{x}_{0}\right) \cap A \cap C_{\mathbf{k}}\right|}{\left|B_{r}\left(\mathbf{x}_{0}\right)\right|} \geq \frac{1}{4}\|\mathbf{k}\| \gamma,
\end{aligned}
$$

thus contradicting (A.2).

Corollary A.4. Let $\Omega \subseteq \mathbb{R}^{n}$ be open, $\mathbf{F}: \mathbb{R} \rightarrow \mathbb{R}^{n}$ a $\mathcal{C}^{1}$ function, $u \in \operatorname{BV}_{\text {loc }}(\Omega)$, and $z \in L_{\text {loc }}^{1}(\Omega)$. Assume that $u$ is a distributional solution to

$$
\operatorname{div} \mathbf{F}(u(\mathbf{x}))=z(\mathbf{x}), \quad \mathbf{x} \in \Omega .
$$

If $u$ is constant on a measurable set $A \subseteq \Omega$, then $z(\mathbf{x})=0$ for a.e. $\mathbf{x} \in A$. 
Proof. By ([2], Thm. 3.96), $\mathbf{F}(u) \in \mathrm{BV}_{l o c}(\Omega)$, and its approximate differential $D \mathbf{F}(u)$ satisfies

$$
D \mathbf{F}(u(\mathbf{x}))=\mathbf{F}^{\prime}(u(\mathbf{x})) \nabla u(\mathbf{x}) \quad \text { for a.e. } \mathbf{x} \in \Omega .
$$

Being, in the assumptions of the corollary, $\operatorname{div} \mathbf{F}(u(\mathbf{x}))$ a measure absolutely continuous w.r.t. the Lebesgue's measure of $\mathbb{R}^{n}$, and since $\nabla u(\mathbf{x})=\mathbf{0}$ for a.e. $\mathbf{x} \in A$ thanks to proposition A.3, we easily get the conclusion.

\section{REFERENCES}

[1] D. Amadori, L. Gosse and G. Guerra, Global BV entropy solutions and uniqueness for hyperbolic systems of balance laws. Arch. Ration. Mech. Anal. 162 (2002) 327-366.

[2] L. Ambrosio, N. Fusco and D. Pallara, Functions of Bounded Variation and Free Discontinuity Problems. Oxford University Press (2000).

[3] F. Ancona and G.M. Coclite, On the attainable set for Temple class systems with boundary controls. SIAM J. Control Optim. 43 (2005) 2166-2190.

[4] F. Ancona and P. Goatin, Uniqueness and stability of $L^{\infty}$ solutions for Temple class systems with boundary and properties of the attainable sets. SIAM J. Math. Anal. 34 (2002) 28-63.

[5] F. Ancona and A. Marson, On the attainable set for scalar nonlinear conservation laws with boundary control. SIAM J. Control Optim. 36 (1998) 290-312.

[6] F. Ancona and A. Marson, Asymptotic Stabilization of Systems of Conservation Laws by Controls Acting at a Single Boundary Point, in Control methods in PDE-dynamical systems. Vol. 426 of Contemp. Math. American Mathematical Society, Providence, RI (2007) 1-43

[7] A. Bressan, Hyperbolic Systems of Conservation Laws. The One-Dimensional Cauchy Problem. Oxford University Press, Oxford (2000).

[8] A. Bressan and G.M. Coclite, On the boundary control of systems of conservation laws. SIAM J. Control Optim. 41 (2002) $607-622$.

[9] J.M. Coron, Control and Nonlinearity. Vol. 136 of Math. Surv. Monogr. American Mathematical Society, Providence, RI (2007).

[10] J.M. Coron, O. Glass and Z. Wang, Exact boundary controllability for 1-D quasilinear hyperbolic systems with a vanishing characteristic speed. SIAM J. Control Optim. 48 (2009/10) 3105-3122.

[11] M. Chapouly, Global controllability of nonviscous and viscous Burgers-type equations. SIAM J. Control Optim. 48 (2009) $1567-1599$

[12] C.M. Dafermos, Generalized characteristic and the structure of solutions of hyperbolic conservation laws. Indiana Math. J. 26 (1977) 1097-1119.

[13] C.M. Dafermos, Hyperbolic Conservation Laws in Continuum Physics, 2nd edition. Springer-Verlag, Berlin (2005).

[14] E. Fernández-Cara and S. Guerrero, Null controllability of the Burgers system with distributed controls. Systems Control Lett. 56 (2007) 366-372.

[15] O. Glass, On the controllability of the 1-D isentropic Euler equation. J. Eur. Math. Soc. 9 (2007) 427-486.

[16] O. Glass, On the controllability of the non-isentropic 1-D Euler equation. J. Differ. Equ. 257 (2014) 638-719.

[17] T. Horsin, On the controllability of the Burgers equation, ESAIM: COCV 3 (1998) 83-95.

[18] H. Holden and N.H. Risebro, Front Tracking for Hyperbolic Conservation Laws. Springer-Verlag, New York (2002).

[19] S.N. Kruzkov, First order quasilinear equations in several independent variables. Math. USSR Sbornik 10 (1970) $217-243$.

[20] M. Léautaud, Uniform controllability of scalar conservation laws in the vanishing viscosity limit. SIAM J. Control Optim. 50 (2012) 1661-1699.

[21] V. Perrollaz, Exact controllability of scalar conservation laws with an additional control in the context of entropy solutions. SIAM J. Control Optim. 50 (2012) 2025-2045.

[22] V. Perrollaz, Asymptotic stabilization of entropy solutions to scalar conservation laws through a stationary feedback law. Ann. Inst. Henri Poincaré, Anal. Non Linéaire 30 (2013) 879-915.

[23] R. Robyr, SBV regularity of entropy solutions for a class of genuinely nonlinear scalar balance laws with non-convex flux function. J. Hyperbolic Differ. Equ. 5 (2008) 449-475. 